\title{
Verankerung des Gleichstellungsgebots auf Europaebene - Fortschritt oder Regression?
}

\section{Beate Rudolf}

„Verankerung des Gleichstellungsgebots auf Europaebene - Fortschritt oder Regression?“ - für den strenggläubigen Europäer ist schon die Fragestellung eine Provokation, ja sogar eine Bedrohung des eigenen Seelenheils. Denn bei einem „Nein“ auf die Frage nach dem Fortschritt droht die europarechtliche Exkommunizierung. Schließlich beruht die Europäische Union auf dem gleichen Dogma wie der Sozialismus: Beide schreiten unaufhörlich voran. Aber da es die Aufgabe der Wissenschaft ist, Dogmen zu hinterfragen, möchte ich versuchen, die mir von den Herausgeberinnen gestellte Frage zu beantworten.

Zunächst werde ich auf die Änderungen im Vertrag über die Europäische Gemeinschaft eingehen, die die Revisionen von Amsterdam und Nizza bewirkt haben. Dabei behandele ich auch die Änderungen im sekundären Gemeinschaftsrecht, speziell der so genannten zweiten Gleichbehandlungsrichtlinie. Anschließend untersuche ich kritisch, ob und wie sich diese Änderungen in der Rechtsprechung des Europäischen Gerichtshofs niedergeschlagen haben. Im Mittelpunkt sollen dabei drei Bereiche stehen: Die Frage nach der Reichweite des Gleichbehandlungsgrundsatzes, die sich im Zusammenhang mit dem Zugang von Frauen zu Berufsstreitkräften gestellt hat, das Verbot der mittelbaren Diskriminierung, das insbesondere bei der Ungleichbehandlung von Vollund Teilzeitarbeitskräften relevant wird, und schließlich die Zulässigkeit von Maßnahmen, durch die Frauen gegenüber Männern bevorzugt werden, Stichwort „Frauenquoten".

Vorweg noch kurz zur Begrifflichkeit: Der Terminus „Gleichstellung“ findet sich im Vertrag über die Europäische Gemeinschaft erst, seitdem im Mai 1999 der Amsterdamer Vertrag ${ }^{1}$ in Kraft getreten ist. Vor diesem Zeitpunkt war im EG-Vertrag nur die Rede vom „Grundsatz des gleichen Entgelts für Männer und Frauen bei gleicher Arbeit". Was ist nun mit „Gleichstellung“ gemeint? Der Begriff „Gleichstellung" betrifft die Lebenswirklichkeit: Es geht um die Durchsetzung der Gleichberechtigung in der Praxis. Diese setzt notwendigerweise die rechtliche Gleichheit voraus. Es fällt auf, dass der EG-Vertrag in anderen Sprachen keinen vergleichbar deutlichen Begriff verwendet. Im Englischen findet sich beispielsweise „,equality“, im Französischen ,égalité“. Daraus lässt sich aber nicht schließen, dass Gleichberechtigung in tatsächlicher Hinsicht nicht gemeint ist. Denn zumindest an einer Stelle, in Art. 141 Abs. 4 EG, verwendet der englische Text für den Begriff der Gleichstellung die Formulierung „,equality in practice“. Vor diesem Hintergrund dürfte sich die Wahl des Terminus „Gleichstellung“ im Deut-

1 Vom 2. 10. 1997, BGBl 1998 II, S. 387, ber. BGBl 1999 II, S. 416, in Kraft getreten am 1. Mai 1999, Bek. v. 6.4.1999, BGBl 1999 II, S. 296. Der Praxis des EuGH folgend werden Bestimmungen des geänderten Vertrages mit „EG“ abgekürzt und solche der Fassung vor „Amsterdam“ mit „EGV“. 
schen durch den Einfluss der Verfassungsentwicklung in Deutschland erklären. Er hat in der Änderung von Art. 3 Abs. 2 GG Aufnahme in die Verfassung gefunden und wird auch in zahlreichen neueren Landesverfassungen verwendet. ${ }^{2}$ Halten wir also fest: Auch der EG-Vertrag bezeichnet mit Gleichstellung eine doppelte Zielsetzung - die Herstellung der rechtlichen und der tatsächlichen Gleichheit von Frauen und Männern.

\section{Das Gleichstellungsgebot im Amsterdamer Vertrag}

Betrachte ich nun die textlichen Änderungen des EG-Vertrages im Hinblick auf die „Gleichstellung von Frau und Mann“, so brachte der Vertrag von Amsterdam diesbezüglich fünf Neuerungen. Sie betreffen die Art. 2, 3, 13, 137 und 141 EG, also die Grundsätze der Gemeinschaft sowie das Kapitel über die Sozialpolitik.

\section{Gleichstellung als Ziel der Gemeinschaft (Art. 2 EG)}

Die erste Neuerung findet sich in Art. 2 EG: Die „Gleichstellung von Männern und Frauen" stellt nunmehr ein Ziel der Gemeinschaft dar. Wie alle anderen Ziele ist auch dieses durch die Errichtung eines Gemeinsamen Marktes und einer Wirtschafts- und Währungsunion sowie - besonders wichtig - durch die Durchführung der gemeinsamen Politiken und Maßnahmen nach den Art. 3 und 4 EG zu fördern. Das bedeutet umgekehrt, dass die Gemeinschaft in den ihr zugewiesenen Bereichen Gleichstellungspolitik durchführen muss. Hierauf werde ich bei Art. 3 EG gleich noch einmal zurückkommen.

Bedeutsam ist das Fehlen einer Begrenzung auf das Wirtschaftsleben, wie es etwa bei dem Ziel einer nachhaltigen Entwicklung der Fall ist. Die Gleichheit der Geschlechter ist also ein Ziel um seiner selbst willen. Es ist damit die einzige genuin grundrechtliche Aufgabe unter dem EG-Vertrag. Verlangt ist die tatsächliche Gleichstellung, denn auch die übrigen Vertragsziele sind auf Verwirklichung in der gesellschaftlichen Realität angelegt. ${ }^{3}$ Was bedeutet es nun in rechtlicher Hinsicht, dass die Gleichstellung ein Ziel der Gemeinschaft bildet? Trotz seiner sehr allgemeinen Fassung ist es für die Gemeinschaftsorgane rechtlich verbindlich. Das hat drei wesentliche Auswirkungen: Die politischen Organe sind verpflichtet, die Gleichstellung bei ihrer Politikgestaltung zu verfolgen. Der EuGH kann und muss sie bei der Auslegung (und Fortbildung) des Gemeinschaftsrechts als Auslegungsmaßstab heranziehen. ${ }^{4}$ Von dem einmal erreichten

2 Art. 10 Abs. 3 S. 2 Berlin, Art. 12 Abs. 3 S. 2 Brandenburg, Art. 13 S. 1 MecklenburgVorpommern, Art. 8 Sachsen, Art. 34 Sachsen-Anhalt und Art. 6 S. 1 Schleswig-Holstein, zitiert nach Pestalozza, Christian (Hrsg.): Verfassungen der deutschen Bundesländer, 6. Aufl. München 1999.

3 v. Bogdandy, Armin: Rdnr. 26 zu Art. 2, in: Grabitz, Eberhard/Hilf, Meinhard (Hrsg.): Das Recht der Europäischen Union, Kommentar (Loseblatt, Stand Januar 2000).

4 So allg. v. Bogdandy, a. a. O., Rdnr. 8 zu Art. 2 und Zuleeg, Manfred: Rdnr. 3 und 7 zu Art. 2, in von der Groeben, Hans/Thiesing, Jochen/Ehlermann, Claus-Dieter (Hrsg.): Kommentar zum EU-/ EG-Vertrag, 5. Aufl. Baden-Baden 1997 (noch zum Maastrichter Vertrag); ausdrücklich für die 
Stand der Verwirklichung von Vertragszielen dürfen keine Abstriche gemacht werden. ${ }^{5}$ Diese Verpflichtungen gelten auch für die Mitgliedstaaten, allerdings nur, soweit sie im Rahmen des EG-Vertrages tätig werden. ${ }^{6}$ Außerhalb dessen wirkt das Vertragsziel „Gleichberechtigung“ für sie nur mittelbar über Art. $10 \mathrm{EG}{ }^{7}$ Danach müssen die Mitgliedstaaten alle Tätigkeiten unterlassen, durch die die Vertragsziele gefährdet werden können. Hingegen begründet Art. 2 EG keine subjektiven Rechte. ${ }^{8}$ Die Norm ist insofern Staatszielbestimmungen im deutschen Verfassungsrecht vergleichbar. Allerdings können Unionsbürger - oder wohl häufiger Unionsbürgerinnen - indirekt von dem Gleichstellungsgebot profitieren, nämlich bei der Auslegung des Gemeinschaftsrechts durch den EuGH. ${ }^{9}$

Mit der Bestimmung der Gleichstellung als Querschnittsaufgabe entsteht das Problem des Konflikts mit anderen Zielvorgaben des EG-Vertrages. Ein Blick in den neuen Art. 2 EG zeigt: Mit dem Amsterdamer Vertrag ist die Anzahl der Ziele der EG von fünf auf zehn angestiegen. Damit nimmt die Gefahr von Zielkonflikten zu. ${ }^{10}$ Wie sind diese zu lösen? Der EuGH gesteht den Gemeinschaftsorganen hier eine weite Einschätzungsprärogative zu. Sie sind lediglich verpflichtet, zwischen den konkurrierenden Zielen Konkordanz herzustellen. " Allerdings bedeutet das nicht, dass alle Ziele in gleichem Maße optimiert werden müssen. Nach Ansicht des Gerichtshofs soll es vielmehr auch zulässig sein, einem Ziel zeitweise den Vorrang einzuräumen. ${ }^{12}$ Dieses Verständnis lässt sich unter der Geltung der Querschnittsklausel für Gleichstellung (und übrigens auch für Umweltschutz, Art. $6 \mathrm{EG}$, sowie für Gesundheitsschutz, Art. 152 Abs. $1 \mathrm{EG}$ ) nicht mehr aufrecht erhalten. Diese Belange dürfen in einer Zielabwägung nicht mehr einfach ,weggewogen“ werden.

$\mathrm{Ob}$ der EuGH den Abwägungsprozess allerdings kontrollieren wird, ist mehr als zweifelhaft: Er hat nämlich eine Untätigkeitsklage für unzulässig erachtet, die das völlige Fehlen einer vertraglich vorgesehenen Politik rügte. ${ }^{13}$ Dies lässt sich meines Erachtens unter der Geltung der Querschnittsklauseln nicht mehr aufrecht erhalten, denn da-

Gleichstellung: Dieball, Heike: Gleichstellung der Geschlechter im Erwerbsleben - neue Vorgaben des EG-Vertrages, EuR 2000, S. 274-284 (277) und Mayer, Ingrid Alice: Gleichberechtigung von Frauen im Aufgaben- und Zielkatalog der EU, djb aktuell 1/1999, S. 15-16 (16).

5 Ukrow, Jörg: Rdnr. 5 zu Art. 2 EG, in Calliess, Christian/Ruffert, Matthias: (Hrsg.), Kommentar zu EU-Vertrag und EG-Vertrag, Neuwied 1999.

6 v. Bogdandy, a. a. O., Rdnr. 52 zu Art. 2 und Ukrow, a. a. O., Rdnr. 27 zu Art. 2 EG.

7 So allg. v. Bogdandy, a. a. O., Rdnr. 8 zu Art. 2, unter Verweis auf Rs. C-265/95 (Kommission ./. Frankreich), Urt. v. 9. 12. 1997, Slg. 1997, I-6959, Rdnr. 24-25, gemeint ist aber wohl Rdnr. 32.

8 Ukrow, a. a. O., Rdnr. 27 zu Art. 2 EG.

9 v. Bogdandy, a. a. O., Rdnr. 9 zu Art. 2.

10 Zur vergleichbaren Problematik im deutschen Arbeits- und Sozialrecht: Scheiwe, Kirsten: Geht die Gleichstellungspolitik im ,Regelungsgestrüpp' des Arbeits- und Sozialrechts unter? Streit 2000, S. 147-151.

11 Zuleeg, a. a. O., Rdnr. 12 zu Art. 2 und Ukrow, a. a. O., Rdnr. 28 zu Art. 2 EG m. Nachw. zur st. Rspr. des EuGH.

12 EuGH, Rs. 5/73 (Balkan Import-Export GmbH /. HZA Berlin-Packhof), Urt. v. 24. 10. 1973, Slg. 1973, S. 1091, Rdnr. 24.

13 So entschieden in Rs. 13/83 (Parlament ./. Rat), Urt. v. 22.5.1985, Slg. 1985, S. 1513, zur Verkehrspolitik. 
mit sind Ziele formuliert, die bei der Abwägung nicht mehr zurückgestellt werden dürfen. Die Entscheidungsprärogative der Gemeinschaftsorgane ist insofern beschränkt. Daher könnte der EuGH jedenfalls evidente Verstöße gegen eines dieser Ziele feststellen. Dies ist auch Verfassungsgerichten bei Staatszielen nach innerstaatlichem Verfassungsrecht möglich. ${ }^{14}$

\section{Gleichstellung als Tätigkeitsbereich der Gemeinschaft (Art. 3 EG)}

Die zweite Änderung betrifft Art. 3 EG. Er umreißt die einzelnen Tätigkeitsfelder der Gemeinschaft und konkretisiert ihre Ziele und Aufgaben. ${ }^{15}$ Neu hinzugekommen ist die Querschnittsklausel in Abs. 2 der Vorschrift. Danach müssen die Gemeinschaften bei allen Tätigkeiten darauf hinwirken, „Ungleichheiten zu beseitigen und die Gleichstellung von Männern und Frauen zu fördern“". Dies verpflichtet die Gemeinschaft auf die Strategie des „Gender Mainstreaming“: ${ }^{16}$ Vor jeder Entscheidung über Politiken und Programme sind demnach die geschlechtsspezifischen Folgen der verschiedenen Vorschläge zu analysieren und bei der Entscheidung zu berücksichtigen. ${ }^{17}$ In der Praxis der Europäischen Kommission wirkt sich das in fünf Schwerpunktbereichen aus: Beschäftigungspolitik, Bildung, Forschung, Strukturfonds und Bekämpfung von Gewalt. ${ }^{18}$

Der Vertrag legt sich nicht auf ein bestimmtes Konzept von Gleichstellung fest. Die Beurteilung, ob eine bestimmte Maßnahme geeignet ist, das Ziel zu erreichen, obliegt den Gemeinschaftsorganen und kann vom EuGH nur unter Beachtung der diesen zustehenden Einschätzungsprärogative überprüft werden. ${ }^{19}$ Allerdings ist es den Gemeinschaftsorganen verboten, hinter dem einmal erreichten Standard, als dem acquis communautaire, zurückzubleiben. Das folgt aus der Funktion von Art. 3 Abs. 2 EG als Verschlechterungsverbot. ${ }^{20}$

\section{Die Ermächtigung zur Diskriminierungsbekämpfung (Art. 13 EG)}

Als dritte Änderung ist Art. 13 EG zu nennen - eine völlig neue Vorschrift, die den Rat ermächtigt, ,geeignete Vorkehrungen zu treffen", um bestimmte Diskriminierungen zu bekämpfen. Angeführt werden zum einen ,herkömmliche“ Diskriminierungsgründe, nämlich Geschlecht, Rasse, ethnische Herkunft, Religion und Weltanschauung, sowie -

14 Sommermann, Kar1-Peter: Staatsziele und Staatszielbestimmungen, Tübingen 1997, S. 437-438.

$15 \mathrm{Zu}$ dieser Funktion siehe v. Bogdandy, a. a. O., Rdnr. 2 zu Art. 3.

16 Dieball, a. a. O., S. 276. Das Gender Mainstreaming in der EU vor dem Amsterdamer Vertrag analysieren Pollack, Mark A./Hafner-Burton, Emilie: Mainstreaming Gender in the European Union, J. of Eur. Public Policy 7 (2000), S. 432-456.

17 Dieball, a. a. O., S. 276, allerdings ohne den Schluss auf eine allgemeine Berücksichtigungspflicht.

18 Siehe etwa "Chancengleichheit für Frauen und Männer in der EU“" (Bericht der Kommission an den Rat, das EP, den Wirtschafts- und Sozialausschuss und den Ausschuss der Regionen), KOM (2000) 123 endgültig (vom 8. 3.2000), S. 6 .

19 Ukrow, a. a. O., Rdnr. 24 zu Art. 3 EG.

20 Allgemein zu dieser Wirkung: Ukrow, a. a. O., Rdnr. $25 \mathrm{zu}$ Art. 3 EG; ihm folgend v. Bogdandy, a. a. O., Rdnr. 21 zu Art. 3. 
erstmals in einem völkerrechtlich verbindlichen Text - Behinderung, Alter und sexuelle Ausrichtung. Der Rat kann aber nur in den Politikbereichen tätig werden, in denen der Gemeinschaft Zuständigkeiten zustehen. ${ }^{21}$ Für die Bekämpfung von Diskriminierung aufgrund des Geschlechts hat Art. 13 EG eine eigenständige Bedeutung nur außerhalb des Arbeits- und Sozialrechts, das durch die Art. 137 und Art. 141 EG gesondert geregelt ist.

Art. 13 EG enthält die Zielsetzung, Diskriminierung zu bekämpfen, aber keine ausdrückliche Verpflichtung zur Förderung der Gleichstellung von Angehörigen diskriminierter Gruppen. Daraus wird zum Teil geschlossen, dass der Rat keine Maßnahmen der positiven Diskriminierung ergreifen oder vorschreiben könne. ${ }^{22}$ Diese Schlussfolgerung vermag aber nicht zu überzeugen: Das Diskriminierungsverbot ist Ausdruck des Gleichheitsgrundsatzes. Versteht man Gleichheit nicht nur als formale, sondern auch als materielle, so kann es - wie bei der Bekämpfung der Diskriminierung der Frau im Arbeitsrecht vom EuGH anerkannt - erforderlich werden, positive Maßnahmen zu ergreifen. ${ }^{23}$ Die Entscheidung über die Erforderlichkeit liegt allein in der Entscheidungsprärogative des Gemeinschaftsgesetzgebers. Der im Rahmen der Vertragsrevision von Nizza angefügten Abs. 2 des Art. 13 EG unterstützt nunmehr diese Auffassung. Danach kann der Rat ,gemeinschaftliche Fördermaßnahmen“ zur Verwirklichung der genannten Zielsetzung erlassen - allerdings unter Ausschluss jeglicher Harmonisierung der Rechts- und Verwaltungsvorschriften der Mitgliedstaaten.

Die rechtliche Qualifizierung von Art. 13 EG wirft Fragen auf: Hat die Norm unmittelbare Wirkung und damit Grundrechtscharakter? Für die Gleichstellung von Mann und Frau ist dies unerheblich, da der EuGH in ständiger Rechtsprechung das Verbot der Diskriminierung aufgrund des Geschlechts als (ungeschriebenes) Gemeinschaftsgrundrecht betrachtet. ${ }^{24}$ Für die übrigen durch Art. 13 EG verbotenen Diskriminierungsgründe gilt das hingegen nicht. Da diese Frage von meinem Thema wegführt, möchte ich dazu hier nur anmerken, dass aus meiner Sicht Art. 13 EG geeignet ist, in der Zukunft unmittelbare Wirkung zu entfalten. Die Wortlautargumentation ist nicht zwingend, wie ein Vergleich mit den beiden Sätzen des Art. 12 EG ergibt, und die entgegenstehende Entstehungsgeschichte verliert mit zunehmender Verankerung der Diskriminierungsverbote auf sekundärrechtlicher Ebene ${ }^{25}$ an Überzeugungskraft.

21 Epiney, Astrid: Rdnr. 5 zu Art. 13, in Calliess, Christian/Ruffert, Matthias (Hrsg.): Kommentar zu EU-Vertrag und EG-Vertrag, Neuwied 1999.

22 Flynn, Leon: The Implications of Article 13 EC - After Amsterdam, Will Some Forms of Discrimination Be More Equal than Others?, CMLRev 36 (1999), S. 1127-1152 (1136-1137).

23 So geschehen in Art. 5 der Richtlinie 2000/43/EG des Rates 29. Juni 2000 zur Anwendung des Gleichbehandlungsgrundsatzes ohne Unterschied der Rasse oder der ethnischen Herkunft, ABl EG L 180/22 vom 19. 7. 2000.

24 Seit Rs. 149/77 (Gabrielle Deftenne ./. Sabena) „Defrenne-III“, Urt. v. 15. 6. 1978, Slg. 1978, 1365, Rdnr. $26 / 29$.

25 Siehe hierzu die Richtlinic 2000/43/EG, a. a. O., sowie die Richtlinie 2000/78/EG des Rates vom 27. November 2000 zur Festlegung eines allgemeinen Rahmens für die Verwirklichung der Gleichbehandlung in Beschäftigung und Beruf, ABl EG L 303/16 vom 2. 12. 2000 (bzgl. Diskriminierung wegen der Religion oder Weltanschauung, einer Behinderung, des Alters oder der sexuellen Ausrichtung). 
Chancengleichheit als Mittel zur Durchsetzung der sozialpolitischen Ziele der Gemeinschaft (Art. 137 EG)

Die letzten beiden Neuerungen sind im Kapitel über die Sozialpolitik enthalten. Der durch den Amsterdamer Vertrag neu gefasste Art. 137 EG inkorporierte das Maastrichter Sozialprotokoll, ${ }^{26}$ das für das Vereinigte Königreich nicht anwendbar war, in den EG-Vertrag. Seit dem bestehen damit wieder für alle Mitgliedstaaten einheitliche Rechtsgrundlagen. Dies wird als „Haupterrungenschaft der Regierungskonferenz [von Amsterdam] im Sozialbereich" gefeiert. ${ }^{27}$ Art. 137 Abs. 1 EG sieht - auch nach der neuerlichen Vertragsrevision von Nizza - bezüglich der "Chancengleichheit von Männern und Frauen auf dem Arbeitsmarkt und Gleichbehandlung am Arbeitsplatz" vor, dass die Gemeinschaft die Tätigkeit der Mitgliedstaaten auf diesem Gebiet „unterstützt und ergänzt". Hierzu überträgt Abs. 2 der Vorschrift dem Rat die Befugnis, durch Richtlinien Mindestvorschriften zu erlassen. ${ }^{28}$ Völlig unklar ist, wie diese Regelung von Art. 141 Abs. 3 EG abzugrenzen ist. Darauf werde ich gleich noch einmal zurückkommen.

Aus der von Art. 137 Abs. 1 EG verwendeten Formulierung, dass die Gemeinschaft die Tätigkeit der Mitgliedstaaten auf dem Gebiet der Chancengleichheit unterstützt, lässt sich schließen, dass auch die Mitgliedstaaten bei ihrer Sozialpolitik auf die Verwirklichung der Gleichstellung verpflichtet sind. Damit geht die Vorschrift noch über Art. 2 EG hinaus. Dieser enthält ja - wie wir bereits gesehen haben - eine solche Verpflichtung nur hinsichtlich der Tätigkeiten der Mitgliedstaaten bei der Anwendung von Gemeinschaftsrecht.

\section{Die Erweiterung des Grundsatzes gleichen Entgelts (Art. 141 EG)}

Die fünfte und letzte Neuerung betrifft den alten Art. $119 \mathrm{EGV}$, also die Vorschrift über den Grundsatz des gleichen Entgelts für Männer und Frauen. Durch „Amsterdam“ wurden mit Art. 141 EG die ersten beiden Absätze neu gefasst; sie enthalten inhaltlich aber nichts Neues. Gleiches Entgelt ist nunmehr ausdrücklich auch bei ,gleichwertiger“ Arbeit geschuldet. ${ }^{29}$ Dieses Gebot hat unmittelbare Wirkung, wie der EuGH schon in der

26 Protokoll Nr. 14 zum Vertrag von Maastricht, geändert durch die Beitrittsakte 1994 vom 24. 6. 1994 (AB1 EG C 241/24 vom 29. 8. 1994) i. d. F. des Beschlusses 95/1/EG, Euratom, EGKS des Rates vom 1. 1. 1995 (ABl EG L 1/1 vom 1. 1. 1995).

27 Thun-Hohenstein, Christoph: Der Amsterdamer Vertrag, Wien 1997, S. 84 (Einfügung durch die Verfasserin).

28 Gemäß Art. 137 Abs. 4 EG bleiben einzelstaatliche - strengere - Gleichstellungsmaßnahmen zulässig, wenn sie im Übrigen mit dem Vertrag vereinbar sind. Unvereinbar ist etwa die Bevorzugung von Frauen bei öffentlicher Auftragsvergabe, die in den Bereich des gemeinschaftsrechtlich geregelten Vergaberechts fällt, welches strikt an dem Kriterium des günstigsten Angebots festhält. Zur Rspr. des EuGH siehe die Nachweise bei Krebber, Sebastian: Rdnr. 24 zu Art. 137, in Calliess, Christian/Ruffert, Matthias (Hrsg.): Kommentar zu EU-Vertrag und EG-Vertrag, Neuwied 1999.

29 Das war bislang nur sekundärrechtlich statuiert, vgl. Art. 1 der Richtlinie 75/117/EWG des Rates vom 10. Februar 1975 zur Angleichung der Rechtsvorschriften der Mitgliedstaaten über die Anwen- 
Vergangenheit geurteilt hat. ${ }^{30}$ Art. 141 Abs. 2 EG stellt klar, dass der Begriff ,Entgelt“ nicht nur den Lohn umfasst, sondern auch alle sonstigen Vergünstigungen, die der Arbeitgeber aufgrund des Dienstverhältnisses direkt oder indirekt an den Arbeitgeber leistet. Auch dies entspricht dem bereits geltenden Sekundärrecht, ${ }^{31}$ der Rechtsprechung des $E u G H^{32}$ und im Übrigen auch völkerrechtlichen Standards. ${ }^{33}$ Darüber hinaus erfuhr Art. $141 \mathrm{EG}$ eine inhaltliche Erweiterung durch seine Absätze 3 und 4. Abs. 3 überträgt dem Rat die Befugnis, „Maßnahmen zur Gewährleistung des Grundsatzes der Chancengleichheit und der Gleichbehandlung von Männern und Frauen in Arbeits- und Beschäftigungsfragen" zu beschließen. Dies erweitert den bisherigen Anwendungsbereich des Gleichstellungsgebotes über den Aspekt des Entgelts hinaus: Es ist nunmehr als allgemeines Gebot zu verstehen, die Gleichstellung der Geschlechter im Arbeitsleben zu fördern. ${ }^{34}$

Problematisch ist das Verhältnis der Vorschrift zu Art. 137 Abs. 2 EG, also der Befugnis zum Erlass von Mindeststandards im Wege der Richtlinie. Denn wie soll man „Maßnahmen zur Gewährleistung des Grundsatzes der Chancengleichheit und der Gleichbehandlung von Männern und Frauen in Arbeits- und Beschäftigungsfragen" (so Art. 141 Abs. 3 EG) abgrenzen von „Chancengleichheit von Männern und Frauen auf dem Arbeitsmarkt und Gleichbehandlung am Arbeitsplatz" (so Art. 137 Abs. 1 EG)? Die erste Formulierung „Arbeits- und Beschäftigungsfragen“ scheint weiter zu reichen als die zweite, die bloß "Arbeitsmarkt" und „Arbeitsplatz“ nennt. Was tun? Muss man überhaupt abgrenzen? Beide Vorschriften ordnen dasselbe Rechtssetzungsverfahren (Mitentscheidungsverfahren nach Art. 251 EG und Anhörung des Wirtschafts- und Sozialausschusses) an. Aber Art. 137 Abs. 2 EG ermächtigt nur zum Erlass von Mindestnormen durch Richtlinien. Also ist eine Abgrenzung notwendig. Natürlich lässt sich die Frage in der Erwartung ignorieren, dass Art. 137 Abs. 2 EG praktisch bedeutungslos wird. ${ }^{35}$ In der Tat hat die Europäische Kommission ihre Änderungsrichtlinie zur zweiten Gleichbehandlungsrichtlinie 76/207/EWG ${ }^{36}$ auf Art. 141 Abs. 3 EG gestützt, ohne Art. 137 Abs. 2 EG überhaupt nur zu erwähnen; Rat und Parlament haben auf dieser

dung des Grundsatzes des gleichen Entgelts für Männer und Frauen, ABl EG L 45/19 vom 19. 2. 1975 .

30 Rs. 61/81 (Kommission ./. Vereinigtes Königreich), Urt. v. 6. 7. 1982, Slg. 1982, 2601, (Rdnr. 7 und 8). Er hat sich dabei auf einen Gleichlauf von Art. 119 EGV und Richtlinie 75/117/EWG, a. a. O., gestützt.

31 Richtlinie 75/117/EWG, a. a. O.

32 Übersicht bei Krebber, a. a. O., Rdnr. 23-35 zu Art. 141.

33 Art. 1 des Übereinkommens Nr. 100 der ILO über die Gleichheit des Entgelts männlicher und weiblicher Arbeitskräfte für gleichwertige Arbeit vom 29. Juni 1951, BGBl 1965 II, S. 24.

34 Oppermann, Thomas: Europarecht, 2. Aufl. München 1999, Rdnr. 1661, ihm folgend Dieball, a. a. O., S. 281 und Thun-Hohenstein, a. a. O., S. 85.

35 So Oppermann, a. a. O., Rdnr. 1661.

36 Richtlinie 76/207/EWG des Rates vom 9. Februar 1976 zur Verwirklichung des Grundsatzes der Gleichbehandlung von Männern und Frauen hinsichtlich des Zugangs zur Beschäftigung, zur Berufsausbildung und zum beruflichen Aufstieg sowie in Bezug auf die Arbeitsbedingungen, ABl EG Nr. L 39/40 vom 14. 2. 1976. 
Rechtsgrundlage die Änderung beschlossen. ${ }^{37}$ Doch praktische Erwägungen können rechtliche nicht ersetzen. Bislang ist in der Literatur kein praktikabler Abgrenzungsvorschlag gemacht worden. ${ }^{38}$ Auch ich habe keine Zauberformel. Ich meine aber, dass Art. 141 Abs. 3 EG seinem Wortlaut nach jedenfalls die speziellere Norm für Regelungen ist, die den Grundsatz des gleichen Entgelts betreffen. Hier kann der Rat „Maßnahmen" beschließen, was (mangels weiterer Beschränkung ${ }^{39}$ ) Rechtsakte gleich welchen Typs - also auch Verordnungen - umfasst. ${ }^{40}$ Problematisch bleibt dann immer noch die Abgrenzung zwischen den übrigen von Art. 141 EG erfassten Bereichen und Art. 137 EG.

Große Aufmerksamkeit hat schließlich der durch „Amsterdam“ geänderte Art. 141 Abs. 4 EG gefunden. Er statuiert, dass der Grundsatz der Gleichbehandlung die Mitgliedstaaten nicht daran hindert, ,zur Erleichterung der Berufstätigkeit des unterrepräsentierten Geschlechts oder zur Verhinderung bzw. zum Ausgleich von Benachteiligungen in der beruflichen Laufbahn spezifische Vergünstigungen beizubehalten oder zu beschließen“. Wie es in einer Erklärung zum Amsterdamer Vertrag heißt, soll diese Vorschriff $^{41}$,in erster Linie der Verbesserung der Lage der Frauen im Arbeitsleben dienen“. ${ }^{42}$ Vom Wortlaut des alten Art. 2 Abs. 4 der zweiten Gleichbehandlungsrichtlinie (Richtlinie 76/207/EWG), der im Streit um die Zulässigkeit von „Frauenquoten“ entscheidend war, unterscheidet sich Art. 141 Abs. 4 EG in mehrfacher Hinsicht. Er erlaubt spezifische Vergünstigungen zur Erleichterung der Berufstätigkeit, aber auch zur Verhinderung oder zum Ausgleich von Benachteiligungen. Demgegenüber durften nach der alten Richtlinienregelung nur die tatsächlich bestehenden Ungleichheiten beseitigt werden. Insbesondere kann der Ausgleich von Benachteiligungen unter Umständen ein Einfallstor bieten, um Frauen für Benachteiligungen in der Vergangenheit zu entschädigen. ${ }^{43}$ In sachlicher Hinsicht dürften beide Klauseln weitgehend identisch sein: Es geht um das gesamte Arbeitsleben, von der Berufsausbildung über den Berufseinstieg bis hin

37 Richtlinic 2002/73/EG des Europäischen Parlaments und des Rates vom 23. September 2002 zur Änderung der Richtlinie 76/207/EWG des Rates zur Verwirklichung des Grundsatzes der Gleichbehandlung von Männern und Frauen hinsichtlich des Zugangs zur Beschäftigung, zur Berufsbildung und zum beruflichen Aufstieg sowie in Bezug auf die Arbeitsbedingungen, ABl EG Nr. L 269/15 vom 5. 10. 2002, vgl. auch dort Erwägungsgrund 5.

38 Siehe etwa den Versuch von Krebber, a. a. O., Rdnr. 97 zu Art. 141, der ihn aber selbst wieder verwirft.

39 Wie etwa in Art. 40 EG: „Der Rat trifft gemäß dem Verfahren des Artikels 251 und nach Anhörung des Wirtschafts- und Sozialausschusses durch Richtlinien und Verordnungen alle erforderlichen Maßnahmen, um (...)“ oder in Art. 150 Abs. 4 EG: ,(...) Maßnahmen“" im Verfahren des Artikels 251 ,unter Ausschluss jeglicher Harmonisierung der Rechts- und Verwaltungsvorschriften“.

40 So zu Recht Bröhmer, Jürgen: Rdnr. 7 zu Art. 57, in Calliess, Christian/Ruffert, Matthias (Hrsg.): Kommentar zu EU-Vertrag und EG-Vertrag, Neuwied 1999.

41 Sie geht auf Art. 6 Abs. 3 des Maastrichter Sozialprotokolls, a. a. O., zurück. Diese Vorschrift betraf nur den Grundsatz des gleichen Entgelts, enthielt keine Verweisung auf die „effektive Gewährleistung der vollen Gleichstellung" und galt nur für Maßnahmen zugunsten von Frauen.

42 Protokollerklärung Nr. 28 des Amsterdamer Rates zu Art. 141 Abs. 4, BGBl 1998 II, S. 448.

43 Ähnlich Fenwick, Helen: From Formal to Substantive Equality: The Place of Affirmative Action in Europe, EPL 4 (1998), S. 507-516 (515), nach deren Ansicht „broad societal disadvantages“" erfasst sein könnten. 
zum beruflichen Aufstieg. Allerdings fehlt in Art. 141 Abs. 4 EG ein ausdrücklicher Verweis auf die soziale Sicherheit. In welchem Verhältnis die beiden Vorschriften zueinander stehen, bedarf der genaueren Klärung. Der EuGH hat im Jahr 2000 in zwei Urteilen, die ich gleich behandeln werde, beide Vorschriften nebeneinander angewendet. Die Kommission war hingegen der Ansicht, Art. 141 Abs. 4 EG habe den alten Art. 2 Abs. 4 der zweiten Gleichbehandlungsrichtlinie (Richtlinie 76/207/EWG) ersetzt und schlug daher vor, die letztgenannte Norm ersatzlos zu streichen. ${ }^{44}$ Hierauf komme ich im thematischen Zusammenhang noch einmal zurück.

\section{Weitere Entwicklungen im Europäischen Recht: Die Gleichbehandlungsrichtlinie, der Vertrag von Nizza und die Charta der Grundrechte}

Inzwischen wurde die zweite Gleichbehandlungsrichtlinie als Richtlinie 2002/73/EG neu gefasst. Sie hebt nunmehr die Pflicht der Mitgliedstaaten hervor, das Ziel der Gleichstellung ,aktiv zu berücksichtigen“; diese Pflicht obliegt ihnen bei ihrer Rechtsetzung und -anwendung sowie ihrer Politikgestaltung im Bereich von Berufszugang, -ausübung und sozialer Sicherheit (Art. 1 Abs. la Richtlinie 2002/73/EG). Damit hat der Begriff der Gleichstellung von Art. 141 Abs. 4 EG seinen Weg ins Sekundärrecht gefunden und betont hier, auf der Ebene der Umsetzung des Gemeinschaftsrechts, ebenfalls die aus dem Gleichheitssatz fließende Notwendigkeit, die Gleichheit von Frauen und Männern auch in der Rechtswirklichkeit zu erreichen.

Eine weitere bedeutende Änderung betrifft die Voraussetzung für die mitgliedstaatliche Befugnis, bestimmte berufliche Tätigkeiten vom Anwendungsbereich der Richtlinie auszunehmen: Es kommt nicht mehr darauf an, ob das Geschlecht für diese Tätigkeit ,auf Grund ihrer Art oder der Bedingungen ihrer Ausübung eine unabdingbare Voraussetzung darstellt", sondern darauf, ob das geschlechtsbezogene Merkmal ,eine wesentliche und entscheidende berufliche Anforderung darstellt, sofern es sich um einen rechtmäßigen Zweck und eine angemessene Anforderung handelt" (Art. 2 Abs. 6 Richtlinie 2002/73/EG). Dies lässt sich als kleiner Fortschritt verstehen, weil nun nicht mehr jedes geschlechtsbezogene Anforderungskriterium es rechtfertigt, eine Diskriminierung zuzulassen, sondern nur Kriterien von herausgehobener Bedeutung für die berufliche Tätigkeit. Zudem lässt sich das Kriterium der ,angemessenen Anforderung“ dahingehend verstehen, dass der mitgliedstaatliche Gestaltungsspielraum einer stärkeren gerichtlichen (Verhältnismäßigkeits-)Kontrolle unterliegen soll. Ob der EuGH diese Gelegenheit nutzt, bleibt abzuwarten. Da aber Art. 9 Abs. 2 der Richtlinie nicht geändert wurde,

44 Vorschlag für eine Richtlinie des Europäischen Parlaments und des Rates vom 7. Juni 2000 zur Änderung der Richtlinie 76/207/EWG des Rates zur Verwirklichung des Grundsatzes der Gleichbehandlung von Männem und Frauen hinsichtlich des Zugangs zur Beschäftigung, zur Berufsausbildung und zum beruflichen Aufstieg sowie in Bezug auf die Arbeitsbedingungen, KOM (2000) 334 endgültig, Dok-Nr. 2000/0142 (COD), 7. Erwägungsgrund, Art. 1 Abs. 3 lit. d und Begründung Rdnr. 30. 
bleibt es bei der Möglichkeit, stereotype Rollenfixierungen in die Bewertung einfließen zu lassen.

Außerdem definiert die Richtlinienänderung - in Übereinstimmung mit den anderen beiden neuen Nichtdiskriminierungsrichtlinien - die Begriffe „unmittelbare" und ,mittelbare Diskriminierung" (Art. 2 Abs. 2 Richtlinie 2002/73/EG). Sie lehnt sich dabei weitgehend an die Rechtsprechung des EuGH an und bringt daher keine Rechtsveränderungen mit sich. Neu und bedeutsam ist hingegen die Einbeziehung von Belästigung und sexueller Belästigung in den Diskriminierungsbegriff (Art. 2 Abs. 3 Richtlinie 2002/73/EG) und deren Definition in Art. 2 Abs. 2 der Richtlinie. Damit reagiert das Europarecht endlich auf diese in der Arbeitswelt häufig anzutreffende Form der Diskriminierung, allerdings in denkbar schwacher Form: (Sexuelle) Belästigung am Arbeitsplatz wird nämlich nur dann einem Arbeitgeber zugerechnet, wenn er selbst handelt oder die Anweisung hierzu gibt (Art. 2 Abs. 4 Richtlinie 2002/73/EG); im Übrigen sind die Mitgliedstaaten nur verpflichtet, die Arbeitgeber zu ,ersuchen“, (sexueller) Belästigung vorzubeugen. Damit bleibt die Bekämpfung dieser Übergriffe entscheidend der Einsicht eines Arbeitgebers überlassen, dass solche Praktiken auch für ihn nachteilig sind, etwa wegen der verringerten Produktivität aufgrund des vergifteten Arbeitsklimas oder wegen negativer Außenwirkung.

Der am 1. Februar 2003 in Kraft getretene Vertrag von Nizza ${ }^{45}$ hat im Bereich der Gleichstellung keine bedeutende Textänderung des EG-Vertrages mit sich gebracht. Insbesondere bleibt das Verhältnis von Art. 137 Abs. 2 EG (jetzt lit. b) zu Art. 141 Abs. 4 EG ungeklärt. Einzige Neuerung ist die Befugnis des Rates, Maßnahmen zur Förderung der Zusammenarbeit zwischen den Mitgliedstaaten anzunehmen, um den Wissens- und Erfahrungsaustausch zwischen diesen zu verbessern (Art. 137 Abs. 2 lit. a EG). Allerdings dürfte der praktische Nutzen dieser Befugnis sehr begrenzt sein; ein Staat, der sich bei den anderen Mitgliedstaaten über Erfolg versprechende Wege zur Gleichstellung informieren wollte, konnte dies auch schon vor der Vertragsänderung.

Schließlich ist noch ein kurzer Blick auf die Charta der Grundrechte der EU ${ }^{46}$ geboten. Das in Art. 21 Abs. 1 der Charta statuierte Diskriminierungsverbot weist einen Katalog verbotener Diskriminierungsgründe auf, der weiter reicht als Art. $13 \mathrm{EG}^{47} \mathrm{Er}$ nennt noch die verbotenen Unterscheidungskriterien Hautfarbe, soziale Herkunft, genetische Merkmale, Sprache, Zugehörigkeit zu einer nationalen Minderheit, Vermögen und Geburt. Da diese Gründe aber nur beispielhaft aufgezählt werden, ist Art. 21 Abs. 1 der Charta als allgemeines Diskriminierungsverbot zu verstehen. Durch beide Eigenschaften geht diese Vorschrift über Art. 13 EG hinaus.

45 Vom 26. 2. 2001, ABl EG C 80/1 vom 10. 3. 2001.

46 Charta der Grundrechte der Europäischen Union vom 7. Dezember 2000, ABl EG C 364/1 vom 18. 12. 2000.

47 „Diskriminierungen insbesondere wegen des Geschlechts, der Rasse, der Hautfarbe, der ethnischen oder sozialen Herkunft, der genetischen Merkmale, der Sprache, der Religion oder der Weltanschauung, der politischen oder sonstigen Anschauung, der Zugehörigkeit zu einer nationalen Minderheit, des Vermögens, der Geburt, einer Behinderung, des Alters oder der sexuellen Ausrichtung sind verboten". 
Sollte die Charta rechtsverbindlich werden - wie dies der Entwurf eines Verfassungsvertrages für die Europäische Union vom Juli 2003 vorsieht $^{48}$ - erübrigt sich die Frage der unmittelbaren Wirkung von Art. 13 EG. Solange sie hingegen nur als feierliche Erklärung verabschiedet worden ist, kann sie von den Gemeinschaftsorganen zumindest als Auslegungshilfe herangezogen werden und so mittelbar wirken. ${ }^{49}$ Für die Gleichstellung von Frauen und Männern formuliert Art. 23 der Charta die Pflicht des Staates, ,die Gleichheit von Männern und Frauen in allen Bereichen, einschließlich der Beschäftigung, der Arbeit und des Arbeitsentgelts“, sicherzustellen. Kritisch anzumerken ist hier, dass der Entwurf ohne Not hinter den Sprachgebrauch des Amsterdamer Vertrages zurückfällt und den Begriff der Gleichstellung nicht verwendet. Das öffnet die Tür für eine restriktive Auslegung.

\section{Europäische Rechtsprechung zu Fragen der Gleichstellung}

Die neuere Rechtsprechung des EuGH nach dem In-Kraft-Treten des Amsterdamer Vertrages hat zu einer Reihe interessanter Urteile und Begründungen im Bereich der unmittelbaren, der mittelbaren und der positiven Diskriminierung geführt, die ich im Hinblick auf die Ausgangsfrage diskutiere.

\subsection{Das Verbot der Diskriminierung aufgrund des Geschlechts}

Im Zusammenhang mit der Frage nach der Reichweite des Verbots der Diskriminierung aufgrund des Geschlechts haben die Entscheidungen des EuGH in den Fällen Angela Sirdar $^{50}$ und Tanja Kreil ${ }^{51}$ heftige Diskussionen ausgelöst, vor allem in Deutschland. Beide betrafen den Ausschluss von Frauen aus den Streitkräften, im ersten Fall nur aus bestimmten Einheiten, im zweiten Fall einen fast generellen Ausschluss. Der Fall Alexander Dory rundet diese Rechtsprechung insofern ab, als nach dem Urteil in der Rechtssache Kreil noch die Frage nach der Vereinbarkeit der Wehrpflicht mit dem Gemeinschaftsrecht offen war, ging es den Klägerinnen Sirdar und Kreil doch um den freiwilligen Zugang zu Beschäftigungsverhältnissen in den nationalen Streitkräften. Die Fälle sind ausgiebig in der Tages- und der Fachpresse behandelt worden. Ich greife daher nur einige, mir wichtig erscheinende Punkte aus der Debatte auf.

48 Siehe Teil II des „Entwurfs eines Vertrages über eine Verfassung für Europa“, vom Europäischen Konvent angenommen am 13. Juni und 10. Juli 2003, Dok. CONV 850/03.

49 Eine gleiche Funktion hatte für den Europäischen Gerichtshof die Gemeinsame Erklärung des Europäischen Parlaments, des Rates und der Kommission vom 5. April 1977, ABl EG C 103/1 vom 27. 4.1977.

50 Rs. C-273/97 (Angela Maria Sirdar ./. The Army Board und Secretary of State for Defence), Urt. v. 26. 10. 1999, Slg. 1999, I-7403.

51 Rs. C-285/98 (Tanja Kreil ./. Bundesrepublik Deutschland), Urt. v. 11. 1. 2000, Slg. 2000, I-69. 


\section{Geltung des Diskriminierungsverbots im Bereich der Streitkräfte?}

Die zentrale Kritik vonseiten der Rechtswissenschaft an den Entscheidungen Sirdar und Kreil richtet sich gegen die Feststellung des Gerichtshofs, dass der Zugang zu den Streitkräften in den Anwendungsbereich des EG-Vertrages falle. ${ }^{52}$ Dabei untersucht der Gerichtshof nur, ob der Vertrag eine allgemeine Ausnahme aus Gründen der öffentlichen Sicherheit anerkenne, ${ }^{53}$ und kommt zu einem negativen Ergebnis. Das konnte nicht überraschen, weil er sich auf ein Urteil aus dem Jahr 1986 stützen konnte. Damals hatte er im Fall Johnston festgestellt, dass die Tätigkeit im Polizeidienst unter die Sozialvorschriften des Gemeinschaftsrechts fiel. ${ }^{54}$ Aber damit konnte der EuGH den grundsätzlichen Einwand nicht wirksam entkräften, dass der Bereich der Verteidigung nicht in die Kompetenzen der Gemeinschaft falle.

Das Gegenargument des Gerichtshofs kommt nur en passant daher. Er verweist darauf, dass der Grundsatz der Gleichbehandlung allgemeine Geltung habe. ${ }^{55}$ Mit anderen Worten, es gibt keine Bereichsausnahmen für den Gleichheitssatz im Sozialrecht. Das drückt eine - für den Europarechtler selbstverständliche - Erkenntnis aus: Soweit die Kompetenzen der Gemeinschaft wirtschaftliche Tätigkeiten betreffen, sind sie Querschnittskompetenzen. Sie können deshalb in Bereiche hineinragen, die im Übrigen der Gemeinschaft entzogen sind. ${ }^{56}$ So hat der EuGH - richtigerweise - schon früher geurteilt, als er Rechtswirkungen des Gemeinschaftsrechts für die Bereiche Sport, Kultur und Bildung bejahte.$^{57}$ Die Besonderheiten einzelner Bereiche vermögen allenfalls Beschränkungen der allgemeinen Regeln zu rechtfertigen, nicht aber ihren völligen Ausschluss. Das gilt für die Notwendigkeit, die Kampffähigkeit einer Armee zu erhalten,

52 Vgl. etwa Arndt, Claus: Waffeneinsatz von Frauen bei der Bundeswehr, NJW 2000, S. 1461-1462 (1461); Doehring, Karl: Vorwärts, Amazonen, RIW 3/2000, Die erste Seite; Dreist, Peter: Das Gebot der Stunde: Streitkräfteöffnung für Frauen, NZWehrR 2000, S. 65-81 (65-66); Eichen, Klaus: Erosion der deutschen Wehrverfassung durch sekundäres Gemeinschaftsrecht?, NZWehrR 2000, S. 45-65 (51-54); Scholz, Rupert: Frauen an die Waffe kraft Europarechts? DÖV 2000, S. 417-420 (419); Stein, Torsten: Anmerkung zu EuGH Urt. v. 11. 1. 2000, EuZW 2000, S. 213-214 (214). Hiergegen schon vor der Entscheidung des EuGH: Zuleeg, Manfred: Frauen in die Bundeswehr, DÖV 1997, S. 1017-1025 (insbes. 1021-1022).

53 Der Gerichtshof verweist insoweit auf den Ausnahmecharakter der Schranken zu einzelnen Grundfreiheiten (etwa Art. 30 S. 1, Art. 39 Abs. 3, Art. 46 Abs. 1 EG), vgl. Rs. Sirdar, a. a. O., Rdnr. 1520 und Rs. Kreil, a. a. O., Rdnr. 15-19. Hiergegen schon BVerwG, NVwZ 1999, S. 1343-1345 (1344-1345).

54 Rs. 222/84 (Marguerite Johnston ./. Chief Constable of the Royal Ulster Constabulary), Urt. v. 15. 5. 1986, Slg. 1986, 1651, Rdnr. 26.

55 Dieses Argument des EuGH übersieht Kämmerer, Jörn Axel: Gleichberechtigung am Gewehr, EuR 2000, S. 102-118 (108-109), bei seiner Kritik an der Heranziehung der Ausnahmebestimmungen des EG-Vertrages, weil der Grundsatz der Gleichbehandlung nur sekundärrechtlich geregelt sei.

56 So auch Stahn, Carsten: Zu den Auswirkungen der EuGH-Urteile Sirdar und Kreil auf das deutsche Recht, EuGRZ 2000, S. 121-135 (126).

57 Vgl. die Nachweise bei Streinz, Rudolf: Frauen an die Front, DVBl 2000, S. 585-595 (589-590). Siehe auch Götz, Volkmar: Anmerkung: Zugang für Frauen zum Dienst in bewaffneten Einheiten, JZ 2000, S. 413-416 (414). 
genauso wie für die Notwendigkeit, die Fußballnationalmannschaft aus eigenen Staatsangehörigen zusammenzusetzen. ${ }^{58}$

So lässt sich die in Deutschland geübte Kritik letztlich auf ein kulturell erklärliches, aber nicht sachlich begründetes Unbehagen zurückführen, Berufssoldaten - oder, wie im Fall Bosman, ${ }^{59}$ Berufssportler - als gewöhnliche Berufstätige anzusehen, ${ }^{60}$ die wegen dieser Eigenschaft in den Regelungsbereich des EG-Vertrages fallen. ${ }^{61}$ Es hat sogar Autoren gegeben, die die Ansicht vertraten, die Bundesregierung hätte den Ausschluss von Frauen aus der Bundeswehr als nationale Grundentscheidung rechtfertigen sollen. Sie sei Teil der nationalen Identität (!). Dann - so diese Meinung - hätte sie sich auf den Schutz der nationalen Identität nach Art. 6 Abs. 3 des Vertrages über die Europäische Union (EU) berufen können. ${ }^{62}$ Die Haltlosigkeit dieses Arguments zeigt schon die Tatsache, dass die Änderung von Art. 12a Abs. 4 S. 2 GG von der Öffentlichkeit fast unbemerkt verabschiedet wurde. ${ }^{63}$

Allerdings ist dem EuGH vorzuwerfen, dass er sich nicht mit der Tatsache auseinander gesetzt hat, ob sich die zweite Gleichbehandlungsrichtlinie nach ihrem Normzweck auf die Streitkräfte der Mitgliedstaaten erstrecken sollte. ${ }^{64}$ Denn das Problem wurde bei Erlass der Richtlinie im Jahre 1976 nicht diskutiert. Das wirft die Frage auf, ob hieraus auf eine Begrenzung ihres Anwendungsbereichs zu schließen ist. Nach meiner Ansicht ist aber zu berücksichtigen, dass sich in den Mitgliedstaaten der Gemeinschaft eine Tendenz weg von der Wehrpflichtarmee und hin zur Berufsarmee zeigt. Armeen werden daher zunehmend als normaler Teil der Exekutive und damit als gewöhnlicher öffentlicher Arbeitgeber angesehen. ${ }^{65}$ Ein derartiger Wandel in den sozialen Anschauungen kann - und muss - die Auslegung des Gemeinschaftsrechts beeinflussen. Gerade solche dynamische Auslegung wird dem Integrationsziel einer ,,immer engeren Union“ (Art. 1 Abs. 2 EU) gerecht. Sie stößt nur an die Grenzen des Wortlauts einer Norm; dieser bereitet aber bei der zweiten Gleichbehandlungsrichtlinie keine Schwierigkeiten.

Von besonderer Bedeutung für die deutsche Rechtsordnung im Zusammenhang mit der Organisation der nationalen Verteidigung ist schließlich das Urteil im Fall Dory, welches die Frage betraf, ob die Beschränkung der Wehrpflicht auf Männer mit der

58 Rs. 36/74 (Walrave und Koch ./. Association Union Cycliste Internationale), Urt. v. 12. 12. 1974, Slg. 1974, S. 1405, Rdnr. 4 und 76.

59 Rs. C-415/93 (Union royale belge des sociétés de football .. Jean-Marc Bosman u. a.), Urt. v. 15. 12. 1995, Slg. 1995, I-4921, Rdnr. 69-73.

60 Sehr deutlich wird das bei Doehring, a. a. O., und Stein, a. a. O., S. 214, die die Frage stellen, ob Kriegsdienst als normales Beschäftigungsverhältnis anzusehen sei, da die typische Konsequenz der Einsatz des eigenen Lebens sei, während die Tätigkeit von Polizisten lediglich gefahrgeneigt sei. Demnach wäre nämlich auch die Tätigkeit als Leibwächter kein Beruf, der in den Anwendungsbereich des EG-Vertrages fällt.

61 Zum Wandel der sozialen Anschauungen als Grund für die „Entdeckung" der zweiten Gleichbehandlungsrichtlinie für den Bereich der Berufsstreitkräfte siehe auch Kämmerer, a. a. O., S. 107108.

62 Stein, a. a. O., S. 213.

63 Gesetz zur Änderung des Grundgesetzes (Art. 12a GG) vom 19. 12. 2000, BGBl 2000 I, S. 1755.

64 So die zutreffende Kritik von Kämmerer, a. a. O., S. 108-109.

65 Kämmerer, a. a. O., S. 108-109. Siehe hierzu auch Stelzenmüller, Constanze: Bürgerin in Uniform, Die Zeit Nr. $3 / 2000$ v. 13. 1. 2000, S. 4. 
zweiten Gleichbehandlungsrichtlinie vereinbar sei. ${ }^{66}$ Der EuGH wiederholt darin seine Feststellung aus den Fällen Sirdar und Kreil, wonach das Militär nicht generell vom Anwendungsbereich der Richtlinie ausgeschlossen sei. ${ }^{67}$ Er nimmt dann aber - im Ergebnis nicht überraschend - den Wehrdienst hiervon aus. Denn dieser zähle zu den „Entscheidungen der Mitgliedstaaten hinsichtlich der militärischen Organisation, die die Verteidigung ihres Hoheitsgebiets oder ihrer unabdingbaren Interessen zum Ziel haben“ und deshalb nicht unter das Gemeinschaftsrecht fielen. ${ }^{68}$ Zwar hätten in die ausschließliche Zuständigkeit der Mitgliedstaaten fallende Entscheidungen über ihre militärische Organisation zwangsläufig Auswirkungen auf den Arbeitsmarkt wie Verzögerungen in der beruflichen Laufbahn der Wehrpflichtigen. Dies genüge jedoch nicht für die Anwendbarkeit des Gemeinschaftsrechts. ${ }^{69}$

Unausgesprochen differenziert der Gerichtshof also zu Recht danach, ob ein gewöhnliches Arbeitsverhältnis - so bei Berufssoldaten - oder ein einseitig-hoheitlich aufgezwungenes Dienstverhältnis besteht. Im ersten Fall kommt die zweite Gleichbehandlungsrichtlinie zur Anwendung, im zweiten Fall ist der Anwendungsbereich des Gemeinschaftsrechts nicht eröffnet. Allerdings ist dieses Abgrenzungskriterium, die Ausgestaltung der militärischen Organisation zur Verteidigung des Staatsgebietes, nicht handhabbar, weil auch die Entscheidung zugunsten einer Berufsarmee eine solche Ausgestaltung ist. Noch schwerer als diese dogmatische Kritik wiegt allerdings ein anderer Umstand: die Tatsache, dass der Gerichtshof die von der deutschen Regierung vorgetragenen Begründungen für die Wehrpflicht in seiner Schlussfolgerung wiedergibt, ohne zu untersuchen, ob sie überhaupt in einem inneren Zusammenhang mit der Differenzierung nach dem Geschlecht stehen. Diese Gründe (Beitrag der Wehrpflicht zur demokratischen Transparenz des Militärapparats, zum nationalen Zusammenhalt, zum engen Kontakt zwischen Streitkräften und Bevölkerung sowie zu der im Verteidigungsfall erforderlichen Mobilmachungsfähigkeit der Streitkräfte) ${ }^{70}$ erklären nämlich nicht, weshalb Frauen von dieser Bürgerpflicht ausgeschlossen werden. Der Gerichtshof erkennt damit implizit einen nicht nachprüfbaren Entscheidungsbereich der Mitgliedstaaten an, welcher sich daraus erklärt, dass eine ausschließliche Kompetenz der Mitgliedstaaten in Frage stand. Dennoch bleibt ein Unbehagen darüber, dass der EuGH sich kritiklos und ohne Notwendigkeit auf eine Position stützt, die Frauen den Rang als gleichwertige Bürgerinnen abspricht und damit stillschweigend alte Rollenklischees perpetuiert.

66 Rs. C-186/01 (Alexander Dory ./. Deutschland), Urt. v. 11. 3. 2003, Slg. 2003, I-2479; dazu Hühn, Uta: Erneut zum Einfluss des Gemeinschaftsrechts auf die Organisation der nationalen Streitkräfte, Verwaltungsrundschau 2003, S. 364-367, die den sachlichen Anwendungsbereich der zweiten Gleichbehandlungsrichtlinie für nicht eröffnet hält (366-367); a. A. schon vor der Entscheidung des EuGH: Zuleeg, Manfred: EuZW 2002, S. 545.

67 Rs. Dory, a. a. O., Rdnr. 34, unter Berufung auf Rs. Sirdar, a. a. O., Rdnr. 28, und Rs. Kreil, a. a. O., Rdnr. 25.

68 Rs. Dory, a. a. O., Rdnr. 35.

69 Rs. Dory, a. a. O., Rdnr. 41.

70 Rs. Dory, a. a. O., Rdnr. 37. 


\section{Die Ausnahme vom Diskriminierungsverbot}

Noch zwei Anmerkungen zur Prüfung des Gerichtshofs, ob der Ausschluss von Frauen aus den Streitkräften eine gerechtfertigte Diskriminierung darstellt:

Nach dem alten Art. 2 Abs. 2 der zweiten Gleichbehandlungsrichtlinie (Richtlinie 76/207/EWG) konnten die Mitgliedstaaten bestimmte berufliche Tätigkeiten von ihrem Anwendungsbereich ausschließen. Davon erfasst waren Tätigkeiten, „für die“ - wie es hieß - „das Geschlecht auf Grund ihrer Art oder der Bedingungen ihrer Ausübung eine unabdingbare Voraussetzung darstellt" ${ }^{\text {" }}{ }^{71}$ Es liegt nun nahe, hierunter nur biologische Unterschiede zwischen den Geschlechtern zu verstehen. So hat es der Generalanwalt im Fall Kreil vorgeschlagen und als Beispiele die Tätigkeit als Tänzer, Mannequin oder Schauspieler genannt. ${ }^{72}$ Der Gerichtshof ist ihm hierin nicht gefolgt, sondern bleibt bei seiner Auslegung, dass die Mitgliedstaaten bei der Festlegung von Ausnahmen einen Gestaltungsspielraum haben. ${ }^{73}$ Damit besteht ein Einfallstor für stereotype Rollenfixierungen fort. Das ist zwar rechtspolitisch bedenklich, ergibt sich aber aus einer systematischen Auslegung der zweiten Gleichbehandlungsrichtlinie. Deren unverändert gebliebener Art. 9 Abs. 2 verpflichtet nämlich die Mitgliedstaaten, „,in regelmäßigen Abständen" die von ihnen ausgeschlossenen beruflichen Tätigkeiten zu überprüfen. Ziel ist es, ,unter Berücksichtigung der sozialen Entwicklung festzustellen, ob es gerechtfertigt ist, die betreffende Ausnahme aufrecht zu erhalten“. Das zeigt, dass die zulässige Ausnahme von Tätigkeiten nicht auf biologischen Unterschieden beruhen muss, sondern auch auf soziale Anschauungen zurückzuführen sein darf. ${ }^{74}$ Insofern wäre es sinnvoll gewesen, die Ausnahmeregelung des Art. 2 Abs. 2 Richtlinie 76/207/EWG bei Änderung der Richtlinie enger zu fassen. Wie gesehen, ist dies mit Art. 2 Abs. 6 Richtlinie 2002/73/EG nur in begrenztem Maße geschehen.

Betrachtet man die Gründe, die die Bundesregierung im Fall Kreil zur Rechtfertigung des Ausschlusses von Frauen vorgebracht hat, so wird eines schnell klar: Der Gerichtshof hatte gar keine andere Wahl, als den Ausschluss für unverhältnismäßig zu halten. Denn die Argumente erweisen sich als fadenscheinig. Ich möchte hier nur das Hauptargument herausgreifen: die erhöhte Schutzbedürftigkeit von Frauen. ${ }^{75}$ Sie ergibt

71 Vgl. dazu die entsprechende Regelung im jetzigen Art. 2 Abs. 6 Richtlinie 2002/73/EG, wonach das ungleich behandelnde, geschlechtsbezogene Merkmal eine ,wesentliche und entscheidende berufliche Anforderung" darstellen muss.

72 Schlussanträge des Generalanwalts Antonio La Pergola vom 26. 10. 1999, Slg. 2000, I-69, Rdnr. 16.

73 Rs. Sirdar, a. a. O., Rdnr. 27, und Rs. Kreil, a. a. O., Rdnr. 24. Im Fall Johnston, a. a. O., Rdnr. 3637 erkannte der Gerichtshof eine Ausnahme für bestimmte Tätigkeiten der Polizei bei schweren inneren Unruhen an. In der ebenfalls in Bezug genommenen Rs. 318/86 (Kommission ./. Frankreich, Urt. v. 30. 6. 1988, Slg. 1988, 3559, Rdnr. 25-27) betr. die Tätigkeit als Aufseher einer Haftanstalt (und damit außerhalb der Frage der öffentlichen Sicherheit) war es hingegen die fehlende Transparenz der Regelung, die zur Europarechtswidrigkeit führte; auf einen Einschätzungsspielraum des Staates kam es daher nicht an.

74 Dies übersieht die - im Übrigen zutreffende - Kritik von Dietlein, Johannes: Anm. zur Entscheidung in der Rs. Tanja Kreil, in: Oetker, Hartmut/Preis, Ulrich: Europäisches Arbeits- und Sozialrecht, Heidelberg (Loseblatt, Stand Dez. 2000), Teil C RL 76/207/EWG, Art. 2, Nr. 15, S. 32-34 (34).

75 Die beiden anderen waren: (1) eine aus der ,leidvollen deutschen Vergangenheit folgende moralische Verpflichtung" und (2) der Notwendigkeit der ,interaktiven Einsatzfähigkeit“" aller kämpfenden 
sich aus Sicht der Bundesregierung zum einen aus Art. 12a Abs. 4 S. 2 GG, der in seiner damals geltenden Fassung Frauen den Dienst mit der Waffe verbot. ${ }^{76}$ Zum anderen entspreche sie dem im alten Art. 2 Abs. 3 der zweiten Gleichbehandlungsrichtlinie Richtlinie niedergelegten Ziel „Schutz der Frau, insbesondere bei Schwangerschaft und Mutterschaft “. ${ }^{77}$ Aber das erste Argument ist ein klassischer Zirkelschluss: Es schließt aus dem Motiv des Verfassungsgebers, Frauen zu schützen, auf deren Schutzwürdigkeit. ${ }^{78}$ Das zweite Argument, wonach Frauen zum Schutz bei Schwangerschaft und Mutterschaft der Zugang zur Bundeswehr verweigert wird, erschöpft sich in dieser Behauptung, ohne ein Wort zur Geeignetheit und Erforderlichkeit des Zugangsverbots zu verlieren. Der in der wissenschaftlichen Literatur unternommene Rettungsversuch ist so verzweifelt, dass ich ihn Thnen nicht vorenthalten möchte: Als Grund wird eine Schutzpflicht des Staates zugunsten des „unerkannten nasciturus”, also des unerkannten Ungeborenen, genannt ${ }^{79}$ - wohl das öffentlich-rechtliche Pendant zum ,unerkannt Geisteskranken" des Zivilrechts. Aber ernsthaft: Dieser Ansatz würde es ermöglichen, Frauen den Zugang zu einem Beruf mit dem Argument zu verwehren, dass jede Frau jederzeit potenziell schwanger ist. Damit würde die Ausnahmeregel, die dem Schutz von berufstätigen Frauen in konkreten Gefahrensituationen dient, in ein Berufsverbot wegen abstrakter und potenzieller Gefährdung umgedeutet. Ein klarerer Verstoß gegen das Verhältnismäßigkeitsprinzip ist kaum denkbar. Aus diesem Grund schied auch diese Ausnahmebestimmung als Rechtfertigungsgrund für den vollständigen Ausschluss von Frauen aus den nationalen Streitkräften aus.

\subsection{Das Verbot der mittelbaren Diskriminierung}

Einen weiteren zentralen Bereich der zweiten Gleichbehandlungsrichtlinie stellt das Verbot der mittelbaren Diskriminierung aufgrund des Geschlechts dar. Dies erfasst Regelungen, die zwar neutral gefasst sind, tatsächlich aber einen wesentlich höheren Anteil von Personen des einen Geschlechts als von Personen des anderen Geschlechts benachteiligen. Weitere Voraussetzung ist, dass die Ungleichbehandlung nicht durch objektive Faktoren gerechtfertigt ist, die unabhängig von einer Diskriminierung aufgrund des Geschlechts sind. ${ }^{80}$ Der EuGH hat mit seiner Rechtsprechung in diesem Be-

Einheiten der Bundeswehr. Vgl. Schlussantrag in der Rs. Kreil, a. a. O., Rdnr. 12-14. Für die beiden letztgenannten Argumente bleibt die Bundesregierung aber die Begründung schuldig. Weder ist erkennbar, dass Frauen im Zweiten Weltkrieg wesentlich mehr gelitten hätten als Männer, schon gar nicht wegen ihrer Zugehörigkeit zur kämpfenden Truppe (siehe auch Kämmerer, a. a. O., S. 114), noch wird erklärt, warum die Tatsache, dass alle Bundeswehrsoldaten im Kriegsfalle überall eingesetzt werden können, unvereinbar mit dem weiblichen Geschlecht sein soll.

76 Sie stützt sich dabei auf die ,Sanitätsdienstentscheidung“ des BVerwG, BVerwGE 103, 301.

77 Jetzt wortgleich geregelt in Art. 2 Abs. 7 Richtlinie 2002/73/EG.

78 Ähnlich Kämmerer, a. a. O., S. 106, der ein „,eindeutiges und überzeugendes Motiv für sein (sc. des Gesetzgebers) legislatorisches Handeln" vermisst.

79 Koch, Christian: Anmerkung zu EuGH, Urt. v. 11. 1. 2000 - C-285/98, DVB1 2000, S. 476-478 (477).

80 Rs. 171/88 (Ingrid Rinner-Kühn /. FWW Spezial-Gebäudereinigung GmbH \& Co. KG), Urt. v. 13. 7. 1989, Slg. 1989, 2743, Rdnr. 12; Rs. C-189/91 (Petra Kirsammer-Hack /. Nurham Sidal), Urt. 
reich vor allem die Rechte teilzeitbeschäftigter Arbeitnehmerinnen erheblich gestärkt, ${ }^{81}$ die europaweit den überwiegenden Anteil an Teilzeitbeschäftigten ausmachen. ${ }^{82}$ An einzelnen Urteilen wurde in der Vergangenheit scharfe Kritik geäußert, vor allem wegen ihrer finanziellen Folgen. Hier scheinen zwei Entscheidungen aus dem Jahr 2000 ein Zurücksteuern des Gerichtshofs anzudeuten (Fälle Jørgensen und Kachelmann), die allerdings durch seine jüngste Rechtsprechung wieder relativiert werden (Fall KutzBauer).

\section{Der Fall Jørgensen}

Die Rechtssache Jørgensen ${ }^{83}$ betraf komplexe Regelungen Dänemarks zur Kostendämpfung im Gesundheitswesen. Das nationale Gericht, das dem EuGH den Rechtsstreit zur Vorabentscheidung vorlegte, kam zu dem Schluss, dass die Regelungen bei einer Gesamtbetrachtung als geschlechtsneutral in Zweck und Wirkung anzusehen seien. Einzelne Teilregelungen würden aber überwiegend Frauen, andere überwiegend Männer treffen. Der EuGH war daher aufgerufen zu entscheiden, ob zur Feststellung einer mittelbaren Diskriminierung eine Gesamtbetrachtung oder ein Vergleich Punkt für Punkt vorzunehmen sei. Der Gerichtshof entschied sich für den Vergleich der einzelnen Bestandteile eines Gesetzes. Er begründet dies damit, dass nur so „echte Transparenz" und damit wirksame Kontrolle gewährleistet seien. Damit verschärft er die dem Staat obliegende Beweislast. Dieser muss nun für jede einzelne Regelung nachweisen, dass die unterschiedliche Behandlung durch objektive Faktoren gerechtfertigt ist. Auf diese Weise dürfte es häufiger zur Feststellung einer mittelbaren Diskriminierung kommen.

Der EuGH konterkariert diesen Fortschritt jedoch sogleich durch eine extensive Anwendung der Rechtfertigungsgründe. Er stellt zwar fest, dass reine Haushaltserwägungen zur Rechtfertigung ungeeignet sind. Denn andernfalls hinge eine grundlegende Regel des Gemeinschaftsrechts - die Gleichheit von Männern und Frauen - vom Zustand der Staatsfinanzen ab. ${ }^{84}$ Anders sei es aber, wenn finanzielle Erwägungen einer sozialpolitischen Entscheidung zugrunde liegen, da den Mitgliedstaaten im Bereich der Sozialpolitik noch ein Gestaltungsspielraum zukomme. Auf den ersten Blick erscheint diese Unterscheidung zwischen bloßen Haushaltserwägungen und der Verfolgung legitimer sozialpolitischer Ziele einleuchtend. In der Praxis dürfte dieser Unterschied hingegen kaum zum Tragen kommen. Beabsichtigte finanzielle Einsparungen lassen sich nämlich in aller Regel damit begründen, dass sie der Aufrechterhaltung von Institutionen der Daseinsvorsorge im Sozialbereich dienen. Außerdem berücksichtigt der Gerichtshof

v. 30. 11. 1993, Slg. 1993, I-6185, Rdnr. 22; Rs. C-226/98 (Birgitte Jørgensen ./. Foreningen af Speciallæger, Sygesikringens Forhandlingsudvalg), Urt. v. 6. 4. 2000 , Slg. 2000, I-2447, Rdnr. 29.

81 Hierzu im Einzelnen Bieback, Karl-Jürgen: Die mittelbare Diskriminierung wegen des Geschlechts, Baden-Baden 1997, insbes. S. 22-113.

82 Siehe auch Kokott, Juliane: Die Gleichstellung von Mann und Frau - Europarecht und deutsches Verfassungsrecht, NJW 1995, S. 1049-1057 (1054-1055).

83 Rs. Jørgensen, a. a. $O$.

84 Hierzu und zum folgenden Rs. Jørgensen, a. a. O., Rdnr. 39-41, unter Berufung auf Rs. C-343/92 (M.A. Roks u. a. ./. Bestuur van de Bedrijfsvereniging voor de Gezondheid, Geestelijke en Maatschappelijke Belangen u. a.), Urt. v. 24. 2. 1994, Slg. 1994, I-571, Rdnr. 35-36. 
nicht, dass gemäß Art. 137 Abs. 1 EG auch die Mitgliedstaaten in ihrer eigenen Sozialpolitik auf die Gleichstellung von Männern und Frauen verpflichtet sind.

\section{Der Fall Kachelmann}

Die Konsequenz aus dem Urteil im Fall Jørgensen ist offensichtlich: Bei der Prüfung der Gemeinschaftsrechtskonformität einer Norm wird es entscheidend, ob die diskriminierende Maßnahme in Bezug auf das verfolgte Ziel auch verhältnismäßig ist. Der EuGH tendiert nun aber dazu, die Verhältnismäßigkeit nicht intensiv zu prüfen. Das zeigt sich sehr deutlich in dem im September 2000 ergangenen Urteil im Fall Kachelmann $^{85}$ zur zweiten Gleichbehandlungsrichtlinie. Der deutsche Fall betraf die betriebsbedingte Entlassung einer teilzeitbeschäftigten Arbeitnehmerin. Sie rügte $\S 1$ Abs. 3 Kündigungsschutzgesetz in der zum Zeitpunkt der Kündigung geltenden Fassung, wonach teilzeitbeschäftigte Arbeitnehmer bei der Sozialauswahl vollzeitbeschäftigten Arbeitnehmern nicht vergleichbar waren. Der EuGH stellt zwar eine faktische Benachteiligung von Teilzeitbeschäftigten gegenüber Vollzeitbeschäftigten fest, da es infolge der geringeren Anzahl von Teilzeitstellen nach einer Entlassung schwerer sei, eine neue Arbeitsstelle zu finden. ${ }^{86}$ Der Gerichtshof sieht aber die Ungleichbehandlung als gerechtfertigt an. Wenn nämlich eine teilzeitbeschäftigte Arbeitnehmerin aus sozialen Gründen gegenüber einem Vollzeitbeschäftigten zu bevorzugen sei, müsse ihr der Arbeitgeber dessen Vollzeitbeschäftigung anbieten. Die Teilzeitbeschäftigte erhält damit so der EuGH - einen Vorteil, der ihr nach innerstaatlichem Recht nicht zusteht. Denn das deutsche Recht kenne keinen Anspruch auf Umsetzung auf einen Vollzeitarbeitsplatz. $\mathrm{Ob}$ er einzuführen sei, könne allein der nationale Gesetzgeber entscheiden. Er entscheide dabei zwischen den widerstreitenden Interessen. ${ }^{87}$

Zentral ist im Urteil des EuGH das Argument, dass nach (damals geltendem) deutschem Recht ein Teilzeitbeschäftigter keinen Anspruch auf Vollzeitbeschäftigung hat. Dies genügt ihm für die Feststellung, dass die Ungleichbehandlung nicht auf dem Geschlecht beruhe. Der Gerichtshof belässt es aber bei der Schlussfolgerung, dass das Ziel des deutschen Gesetzgebers legitim gewesen sei. Er prüft hingegen nicht, ob dieses Ziel auch mit einem verhältnismäßigen Mittel verfolgt wird. Ein milderes Mittel wäre beispielsweise zunächst eine Vergleichbarkeit von Teil- und Vollzeittätigkeit bei der Sozialauswahl anzunehmen. Sprechen die Kriterien zugunsten des Teilzeitbeschäftigten, müsste der Arbeitgeber dem unterlegenen Vollzeitbeschäftigten im Wege der Änderungskündigung eine Teilzeitstelle anbieten. Das hätte den positiven Nebeneffekt, dass Arbeitgeber stärker dazu gezwungen würden, über die Teilbarkeit von Stellen nachzudenken. Das würde zudem - gesamtgesellschaftlich gesehen - die Erfolgschancen von Frauen bei der Arbeitsuche verbessern. Bedenkt man dann noch, dass unter der Geltung des Art. 137 Abs. 1 EG auch die Mitgliedstaaten an das Gleichstellungsgebot gebunden

85 Rs. C-322/98 (Bärbel Kachelmann /. Bankhaus Hermann Lampe KG), Urt. v. 26.9. 2000, Slg. 2000 , I-7505.

86 Rs. Kachelmann, a. a. O., Rdnr. 27.

87 Rs. Kachelmann, a. a. O., Rdnr. 33-34. 
sind, so würde durch diese Lösung auch nicht deren sozialpolitischer Entscheidungsspielraum in unzulässiger Weise beschränkt werden.

Zwar ist der Entscheidungsausspruch des EuGH infolge der Änderung des Beschäftigungsförderungsgesetzes heute nicht mehr relevant. ${ }^{88}$ Dies ändert aber nichts an der Kritikwürdigkeit seiner Vorgehensweise, unter Berufung auf den sozialpolitischen Entscheidungsspielraum der Mitgliedstaaten selbst unverhältnismäßige Maßnahmen als gerechtfertigte Diskriminierungen zuzulassen.

\section{Der Fall Kutz-Bauer}

Mit der Frage des sozialpolitischen Gestaltungsspielraums der Mitgliedstaaten beschäftigte sich eine weitere wichtige Entscheidung des EuGH, die zu einer deutschen tarifvertraglichen Regelung erging. Die fragliche Vorschrift gestaltete die Berechtigung, Altersteilzeit in Anspruch zu nehmen, für Männer und Frauen unterschiedlich aus, indem sie diese mit dem Zeitpunkt enden ließ, in dem erstmals eine Rente aus der gesetzlichen Altersversorgung bezogen werden kann. ${ }^{89}$ Der Gerichtshof erklärte im Fall KutzBauer diese Regelung als mittelbare Diskriminierung für unvereinbar mit der zweiten Gleichbehandlungsrichtlinie, ${ }^{90} \mathrm{da}$ das Recht, ab dem 60. Lebensjahr Altersteilzeit zu vereinbaren, demzufolge fast ausschließlich Männern zusteht. Er wies dabei die zur Rechtfertigung vorgebrachten Gründe zurück, wonach die Regelung Einstellungen fördern solle und die Erstreckung der Altersgrenze von 65 Jahren auch auf Frauen die Rentenkassen übermäßig belasten würde. Dabei wiederholte der EuGH nicht nur seine Feststellungen aus dem Fall Jørgensen, wonach bloße haushaltspolitische Erwägungen nicht ausreichen. $^{91}$ Bedeutsam ist vielmehr, dass der Gerichtshof den sozialpolitischen Entscheidungsspielraum der Mitgliedstaaten enger zieht, indem er dem Staat die Beweislast dafür auferlegt, dass die unterschiedliche Behandlung durch objektive, nicht geschlechtsbezogene Faktoren gerechtfertigt ist. ${ }^{92}$ Hierdurch begegnet er der im Fall Jørgensen offenbar gewordenen Gefahr, dass Haushaltserwägungen hinter nicht nachprüfbaren sozialpolitischen Begründungen versteckt werden.

$88 \S 2$ Abs. 1 des Gesetzes über arbeitsrechtliche Vorschriften zur Beschäftigungsförderung, BGB1 1985 I, S. 710, i. d. F. des Änderungsgesetzes v. 25. 9. 1996, BGB1 1996 I, S. 1976. Dies wirkt sich auch auf Kündigungen aus, vgl. Preis, Ulrich: Rdnr. $4 \mathrm{zu} \& 2$ BeschFG, in: Ascheid, Reiner/Preis, Ulrich/Schmidt, Ingrid (Hrsg.): Großkommentar zum Kündigungsrecht, München 2000, und Kiel, Heinrich: Rdnr. $686 \mathrm{zu} \S 1 \mathrm{KSchG}$, ibid.

89 Rs. C-187/00 (Helga Kutz-Bauer ./. Freie und Hansestadt Hamburg), Urt. v. 20. 3. 2003, Slg. 2003, I-2741.

90 Eine Anwendbarkeit der Richtlinie 79/7/EWG des Rates vom 19.12. 1978 zur schrittweisen Verwirklichung des Grundsatzes der Gleichbehandlung von Männern und Frauen im Bereich der sozialen Sicherheit (ABl EG L 6/24 v. 10. 1. 1979) lehnte der EuGH mit der Begründung ab, Altersteilzeit betreffe die Arbeitsbedingungen und falle daher unter die zweite Gleichbehandlungsrichtlinie, Rs. C-187/00, a. a. O., Rdnr. 44-45.

91 Vgl. dazu auch jüngst Rs. C-77/02 (Erika Steinicke ./. Bundesanstalt für Arbeit), Urt. v. 11. 9. 2003.

92 Rs. Kutz-Bauer, a. a. O., Rdnr. 62. So auch Streinz, Rudolf: Anm. zu Rs. Kutz-Bauer, JuS 2003, S. $806(808)$. 


\subsection{Frauenförderprogramme und "Positive Diskriminierung“}

Als letzten Bereich möchte ich die neuere Rechtsprechung des EuGH zu Frauenförderprogrammen beleuchten, insbesondere zur so genannten „Frauenquote“. Die hier grundlegenden Entscheidungen in den Fällen Kalanke und Marschall dürften allgemein bekannt sein. Der Fall Badeck ist insbesondere im Hinblick auf den Begriff der gleichen Qualifikation und das Verhältnis von Entscheidungskriterien in Pattsituationen bedeutsam. Der Fall Abrahamsson hingegen bezieht sich auf konkrete Maßnahmen zur Frauenförderung durch Einrichtung entsprechend bestimmter Stellen und damit auf ein anderes Mittel der positiven Diskriminierung.

\section{Von Kalanke zu Marschall}

Zur Erinnerung: Der Fall Kalanke ${ }^{93}$ betraf eine bremische Regelung, nach der im Fall der Unterrepräsentation von Frauen die Bewerberin gegenüber einem gleich qualifizierten männlichen Mitbewerber stets zu bevorzugen war. Der EuGH erklärte dies für unvereinbar mit der zweiten Gleichbehandlungsrichtlinie. Hierfür nannte er zwei Argumente. Erstens: Der absolute und unbedingte Vorrang von Frauen gehe über eine Förderung der Chancengleichheit hinaus und überschreite damit die Grenzen der in dem damaligen Art. 2 Abs. 4 der Richtlinie zugelassenen Ausnahme. Zweitens liege ein Verstoß darin, dass die Zielsetzung der Regelung, auf allen Funktionsebenen eine gleiche Anzahl von Frauen und Männern zu erreichen, an die Stelle der Chancengleichheit die Ergebnisgleichheit setze. ${ }^{94}$ Diese doppelte Abstützung der Entscheidung führte zu Spekulationen darüber, ob auch Frauenquoten mit „Öffnungsklausel“ europarechtswidrig seien. ${ }^{95}$ Dabei handelt es sich um Klauseln, nach der eine Bevorzugung der Bewerberin unterbleibt, wenn die Person des männlichen Mitbewerbers betreffende Gründe überwiegen. Hierüber hatte der EuGH im Fall Marschall ${ }^{96} \mathrm{zu}$ entscheiden. Der Gerichtshof verabschiedete sich von dem zweiten Argument aus der Kalanke-Entscheidung und bezeichnete den Automatismus der Bevorzugung von Frauen als entscheidend. Er erklärte folglich die Klausel für europarechtskonform. ${ }^{97}$

Neu sind die Ausführungen des Gerichtshofs, dass auch bei gleicher Qualifikation eine Bewerberin nicht die gleichen Chancen wie ein Bewerber habe. Stereotype Rollen-

93 Rs. 450/93 (Eduard Kalanke ./. Freie Hansestadt Bremen), Urt. v. 17. 10. 1995, Slg. 1995, I-3051.

94 Rs. Kalanke, a. a. O., Rdnr. 22-23. Hiergegen etwa Sacha-Prechal, Casenote: Kalanke v. Freie Hansestadt Bremen, CMLRev. 33 (996), S. 1245-1259 (1257) und Veldman, Albertine: The Lawfulness of Women's Priority Rule in the EC Labour Market, Maastricht J. Eur. \& Comp. L. 5 (1998), S. 403-414 (405), da Ergebnisgleichheit nur dann Chancengleichheit ersetze, wenn eine Frau auch einem besser qualifizierten Mann vorgezogen werden könne, bis gleiche Repräsentation erreicht sei.

95 Eine gute Übersicht über die verschiedenen Schlussfolgerungen aus der Entscheidung in der deutschsprachigen Literatur gibt Suhr, Oliver: Grenzen der Gleichbehandlung: Zur Vereinbarkeit von Frauenquoten mit dem Gemeinschaftsrecht, EuGRZ 1998, S. 121-128 (123 und dort Fn. 23 und 24). Siehe auch More, Gillian: Annotation: Hellmut Marschall v. Land Nordrhein-Westfalen, CMLRev. 36 (1999), S. 443-452 (448-449).

96 Rs. C-409/95 (Hellmut Marschall ./. Land Nordrhein-Westfalen), Urt. v. 11. 11. 1997, Slg. 1997, I6363.

97 Rs. Marschall, a. a. O., Rdnr. 32-33. 
vorstellungen führten $\mathrm{zu}$ einer Tendenz, männliche Bewerber $\mathrm{zu}$ bevorzugen. ${ }^{98}$ Damit kommt der Gerichtshof von einem rein formalen Verständnis der Gleichheit ab. Er erkennt an, dass die zweite Gleichbehandlungsrichtlinie auch materielle Gleichheit anstrebt. ${ }^{99}$ Dafür müssen die sozialen Realitäten einbezogen und tatsächliche Hindernisse für die Gleichheit beseitigt werden. Vor diesem Hintergrund wurden die beiden für das Jahr 2000 erwarteten Entscheidungen des EuGH über Maßnahmen der Frauenförderung mit Spannung erwartet.

\section{Der Fall Badeck}

Die Rechtssache Badeck ${ }^{100}$ betraf verschiedene Regelungen des hessischen Gleichstellungsgesetzes ${ }^{101}$. Von den zahlreichen Einzelfragen greife ich nur die wichtigste heraus. Diese stellt sich im Zusammenhang mit $\S 10$ Abs. 1 und 3 HessGlG: Danach sind bei der Bestimmung der Qualifikation diejenigen Fähigkeiten und Erfahrungen, die durch Familienarbeit erworben wurden, zu berücksichtigen, soweit sie für die Eignung, Leistung und Befähigung der Bewerberinnen und Bewerber bedeutsam sind. Dienstalter, Lebensalter und der Zeitpunkt der letzten Beförderung dürfen nur insoweit berücksichtigt werden, als sie ebenfalls für die Eignung, Leistung und Befähigung von Bedeutung sind. Ausdrücklich ist angeordnet, dass Familienstand und Einkommen des Partners nicht berücksichtigt werden dürfen. Teilzeitbeschäftigungen, Beurlaubungen und Verzögerungen aufgrund von Kinder- oder Angehörigenbetreuung dürfen sich nicht negativ auswirken. Mit anderen Worten: Der Gesetzgeber hat versucht, Kriterien auszuscheiden, die bei traditioneller Rollenverteilung Männem zugute kommen, und diejenigen positiv umzudeuten und einzubeziehen, die in der Vergangenheit Frauen zum Nachteil gereicht haben. Mit Hinweis auf die Marschall-Entscheidung kommt der EuGH kurzerhand zu dem Ergebnis, dass solche Kriterien die materielle, nicht nur formale Gleichheit herbeiführen wollten. Sie verringerten die in der sozialen Wirklichkeit auftretenden faktischen Ungleichheiten. Deshalb sei es unerheblich, dass diese Kriterien, obwohl geschlechtsneutral formuliert, in der Regel Frauen zugute kämen. ${ }^{102}$

Ergibt sich nun bei Anwendung dieser Kriterien ein "qualifikatorisches Patt", so muss bei Unterrepräsentation von Frauen im betreffenden Bereich zugunsten der Frau entschieden werden, wenn keine Gründe von größerem rechtlichen Gewicht entgegenstehen. Diese Einschränkung ist freilich nicht im HessGlG enthalten. Sie hat der Hessi-

98 Rs. Marschall, a. a. O., Rdnr. 29-30. Zu Recht weisen More, a. a. O., S. 450, und Veldman, a. a. O., S. 410, darauf hin, dass der Gerichtshof mit der Berücksichtigung der tatsächlichen Auswirkung scheinbar nicht diskriminierender Maßnahmen sich wieder seiner sonstigen Rechtsprechung zum Diskriminierungsverbot annähert.

99 So auch Fenwick, a. a. O., S. 514, Mancini, G. F./O'Leary, S.: The New Frontiers of Sex Equality Law in the European Union, Eur. L. Rev. 24 (1999), S. 331-353 (336), und More, a. a. O., S. 449450.

100 Rs. C-158/97 (Georg Badeck u. a. ./. Hessischer Ministerpräsident und Landesanwalt beim Staatsgerichtshof des Landes Hessen), Urt. v. 28. 3. 2000, Slg. 2000, I-1875.

101 Vom 21. 12. 1993, GVB1. I, S. 729, geändert durch Art. 4 des Siebten Dienstrechts-ÄndG vom 7. 7. 1998, GVBl. I, S. 260 und Art. 2 des G z. Beschleunigung von Entscheidungsprozessen innerhalb der öff. Verw. vom 6. 7. 1999, GVBl. I, S. 338.

102 Rs. Badeck, a. a. O., Rdnr. 32. 
sche Staatsgerichtshof - mit Blick auf Art. 3 Abs. 2 GG und auf die MarschallEntscheidung - in das Gesetz hineingelesen. ${ }^{103}$ Welche Gründe können die Frauenförderung überwiegen? Nach Auskunft des Hessischen Ministerpräsidenten sind dies folgende fünf Fallgruppen, die auf sozialen Erwägungen beruhen: eine frühere Tätigkeit im öffentlichen Dienst, die wegen Familienarbeit beendet wurde, eine bisherige Teilzeittätigkeit aufgrund von Familienarbeit, die nunmehr in Vollzeit ausgeübt werden soll, ehemalige Zeitsoldaten, Schwerbehinderte, Langzeitarbeitslose. ${ }^{104}$

Der EuGH sieht diese ungeschriebenen Kriterien als ausreichend an, um zu dem Schluss zu kommen, dass die hessische Regelung keinen absoluten Vorrang der weiblichen Bewerber begründe. Für ihn ist es anscheinend unerheblich, dass andere Kriterien, die in der Person des (männlichen) Bewerbers begründet sind, nicht berücksichtigt werden können. Die noch in der Marschall-Entscheidung genannte Notwendigkeit der Berücksichtigung des Einzelfalles reduziert sich damit beträchtlich. Denn die ersten beiden Ausnahmetatbestände - Ausscheiden aus dem öffentlichen Dienst wegen Familienarbeit oder Teilzeittätigkeit aus diesem Grund - werden in aller Regel Frauen zugute kommen. Damit bleiben nur noch seltene Fallgruppen (ehemalige Zeitsoldaten, Langzeitarbeitslose, Schwerbehinderte). Die ersten beiden hiervon dürften ihrer Zielsetzung nach nur bei Neueinstellungen zum Tragen kommen, nicht aber bei Beförderungen. Damit nähert sich die Regelung einer absoluten - und damit nach der Kalanke-Rechtsprechung des EuGH verbotenen - Vorrangklausel sehr weitgehend an. Insofern ist die Position des Gerichtshofs eine rein formale: Sobald auch nur eine - theoretische - Möglichkeit besteht, dass ein Mann einer gleich qualifizierten Frau vorgezogen werden kann, ist die Vorrangklausel nicht mehr absolut und daher europarechtlich zulässig. Auf die Notwendigkeit einer individuellen Prüfung der persönlichen Umstände der Bewerber verzichtet der EuGH im Gegensatz zur Marschall-Entscheidung ganz.

Einen weiteren Punkt möchte ich nur ganz kurz anreißen: Der EuGH hatte auch über $\S 9$ HessGlG zu befinden, wonach alle Bewerberinnen, mindestens aber ebenso viele Frauen wie Männer, zum Vorstellungsgespräch einzuladen sind. Einzige Bedingung ist, dass sie die gesetzlichen Voraussetzungen für die ausgeschriebene Stelle erfüllen. Generalanwalt und Gerichtshof sind hier übereinstimmend der Ansicht, dass die Vorschrift die Chancengleichheit von gleich qualifizierten Frauen und Männern fördere und kein bestimmtes Resultat vorschreibe. ${ }^{105}$ Denn sie bietet qualifizierten Bewerberinnen zusätzliche Möglichkeiten, in die Arbeitswelt einzutreten oder aufzusteigen. Chancengleichheit bedeutet demnach für EuGH und Generalanwalt nicht bloß die für eine Bewerberin theoretisch bestehende Möglichkeit, zu einem Vorstellungsgespräch eingeladen zu werden, sondern erst die tatsächliche Einladung. Damit legen Gerichtshof und Generalanwalt auch hier ein materielles Verständnis von Gleichheit zugrunde.

103 Vgl. Rdnr. 36 der Schlussanträge des Generalanwalts Antonio Saggio v. 10. 6. 1999, Slg. 2000, I1875.

104 So die Auskunft des Hessischen Ministerpräsidenten, wiedergegeben im Urteil Rs. Badeck, a. a. O., Rdnr. 34.

105 Urteil Rs. Badeck, a. a. O., Rdnr. 60-62 und Schlussantrag Saggio, a. a. O., Rdnr. 41. 


\section{Der Fall Abrahamsson}

Die Rechtssache Abrahamsson ${ }^{106}$ betraf die Vereinbarkeit schwedischer Maßnahmen zur Erhöhung des Frauenanteils am Lehrpersonal der staatlichen Hochschulen. Die erstmalige Besetzung bestimmter neu einzurichtender Stellen sollte nach folgender $\mathrm{Re}-$ gel erfolgen: Eine hinreichend qualifizierte Bewerberin war einem Mann vorzuziehen, selbst wenn dieser besser qualifiziert war. Hierfür galt allerdings eine Grenze: Die Bevorzugung hatte zu unterbleiben, wenn der Qualifikationsunterschied derart groß war, dass die Bevorzugung der Frau gegen das Erfordernis der Sachgerechtigkeit verstieße.

Überraschend ist das Schweigen des EuGH zu der Tatsache, dass die Regelung nicht die gleiche Qualifikation der beteiligten Bewerber voraussetzt. ${ }^{107}$ Stattdessen zieht er wieder die zwei Kriterien aus der Marschall-Entscheidung heran: kein Automatismus der Frauenbevorzugung und Pflicht zur Berücksichtigung der persönlichen Situation der Bewerber. Schon am Fehlen dieser Individualprüfung hätte er die schwedische Regelung scheitern lassen können. ${ }^{108}$ Er konstatiert jedoch noch zusätzlich eine automatische Bevorzugung von Frauen, da die Reichweite der Ausnahmebestimmung unklar sei. ${ }^{109}$

Dieses Bemühen um Konsistenz der Rechtsprechung ist anerkennenswert. Jedoch kann die Argumentation des Gerichtshofs nicht überzeugen. Ebenso gut kann sich die Unklarheit der Entscheidungskriterien zu Lasten von Bewerberinnen auswirken. Schließlich hat es das - in aller Regel überwiegend männlich besetzte - Entscheidungsgremium in der Hand, einen männlichen Mitbewerber als so viel höher qualifiziert anzusehen, dass die Ausnahmeregel greift. Gerade durch die offene Formulierung der Ausnahmevorschrift sind dem Missbrauch hier Tür und Tor geöffnet. Insbesondere können auf diesem Wege wieder Rollenstereotype in die Entscheidung einfließen. Überzeugender wäre es daher gewesen, wenn der EuGH sich nicht krampfhaft an die Kriterien aus der Marschall-Entscheidung gehalten hätte. Stattdessen hätte er besser den rechtsstaatlichen Bestimmtheitsgrundsatz herangezogen, um die Regelung scheitern zu lassen.

Auf eine weitere Vorlagefrage des nationalen Gerichts stellt der EuGH fest, dass eine Regelung auch dann europarechtswidrig bleibt, wenn sie sich nur auf eine bestimmte Anzahl neuer Stellen bezieht. Sein Argument: Die Diskriminierung behalte ihren absoluten und unverhältnismäßigen Charakter. ${ }^{110}$ Aber ist das nicht ein Zirkelschluss? Es fragt sich doch gerade, ob der enge Anwendungsbereich die Regelung nicht verhältnismäßig werden lässt. Entscheidend ist also, ob man - wie der Gerichtshof - nur die konkrete Maßnahme betrachtet, oder ob man sie in ihrem rechtlichen und tatsächlichen Kontext sieht. Für den letztgenannten Ansatz spricht das Argument, das der EuGH in der Marschall-Entscheidung betont hat, dass formale Gleichheit oft nicht ausreicht, um

106 Rs. C-407/98 (Katarina Abrahamsson und Leif Anderson ./. Elisabet Foglqvist), Urt. v. 6. 7. 2000, Slg. 2000 , I-5539.

107 Auf diese weist auch der EuGH ausdrücklich hin, Rs. Abrahamsson, a. a. O., Rdnr. 45.

108 Rs. Abrahamsson, a. a. O., Rdnr. 52-53.

109 Rs. Abrahamsson, a.a. O., Rdnr. 49. Noch schärfer Generalanwalt Antonio Saggio in seinen Schlussanträgen v. 16. 11. 1999, Slg. 2000, I-5539, Rdnr. 28, der kritisiert, die Beurteilung der Qualifikation stehe somit nur an zweiter Stelle, „dénaturant complètement (...) le rôle de la sélection“.

110 Rs. Abrahamsson, a. a. O., Rdnr. 58-59. 
zu verhindern, dass stereotype Rollenvorstellungen zu Entscheidungen zu Lasten von Frauen führen.

Der Gerichtshof kommt also im Fall Abrahamsson zu dem Ergebnis, dass die schwedische Regelung gegen den (damaligen) Art. 2 Abs. 4 der zweiten Gleichbehandlungsrichtlinie (76/207/EWG) verstößt. Folglich prüft er, ob sie denn mit Art. 141 Abs. 4 EG vereinbar ist. Diese Frage verneint er mit dem knappen Argument, dass auch das Primärrecht keine unverhältnismäßige Auswahlmethode zulasse. ${ }^{11}$

\section{Zum Verhältnis von EG-Vertrag und abgeleitetem Recht aus Sicht der Europäi- schen Rechtsprechung: Ein Fazit}

Die Analyse des Urteils im Fall Abrahamsson führte zu der Frage des Verhältnisses von primär- und sekundärrechtlichen Ermächtigungen zurück, die ich bereits oben aufgeworfen habe. Für den Gerichtshof ist das Vorgehen im Fall Abrahamsson klar: Er legt die zweite Gleichbehandlungsrichtlinie im Lichte seiner früheren Rechtsprechung aus. Da sie abgeleitetes Recht ist, prüft er sie anschließend auf ihre Vereinbarkeit mit dem Primärrecht. Wichtiger noch als das, was der EuGH in seiner Entscheidung Abrahamsson sagt, ist freilich, was er nicht sagt. Der Generalanwalt hatte vorgeschlagen, die Richtlinie im Lichte des geänderten Art. 141 Abs. 4 EG auszulegen. ${ }^{12}$ Nach seiner Ansicht spiegelt diese Vorschrift ein neues Verständnis des Gleichbehandlungsgebotes wider: Maßnahmen zur Beseitigung der Ungleichheit sind grundsätzlich zulässig. Einzige Voraussetzung: Sie müssen eine Berücksichtigung der individuellen Umstände vorsehen. Folge dieses Ansatzes ist, dass Maßnahmen der positiven Diskriminierung nicht länger als Ausnahme vom Gleichheitssatz anzusehen sind. Sie dienen vielmehr seiner Verwirklichung. Weitere - unausgesprochene - Konsequenz ist, dass Art. 141 Abs. $4 \mathrm{EG}$ - anders als vom EuGH vertreten - nicht restriktiv auszulegen ist.

Eine letzte Überlegung gilt den erlaubten Ausgleichsmaßnahmen zugunsten des unterrepräsentierten Geschlechts. Hier ist ein Urteil hervorzuheben, das über seinen Gegenstand hinaus - die Berechnung des rentenfähigen Dienstalters für Beamtenpensionen nach französischem Recht - von allgemeiner Bedeutung ist. ${ }^{113}$ Es lässt nämlich erkennen, dass der Gerichtshof auch die Befugnis der Mitgliedstaaten, zugunsten des unterrepräsentierten Geschlechts spezifische Vergünstigungen beizubehalten oder zu beschließen, welche Benachteiligungen in der beruflichen Laufbahn ausgleichen sollen,

111 Rs. Abrahamsson, a. a. O., Rdnr. 40.

112 Schlussanträge Rs. Abrahamsson, a. a. O., Rdnr. 26-28. In diese Richtung weist nun auch die Neuregelung der fraglichen Richtlinienbestimmung in Art. 2 Abs. 8 Richtlinie 2002/73/EG: Danach sind die Mitgliedstaaten berechtigt, ,im Hinblick auf die Gewährleistung der vollen Gleichstellung von Männern und Frauen Maßnahmen im Sinne von Artikel 141 Absatz 4 des Vertrages [gemeint ist der EG-Vertrag] bei[zu]behalten oder [zu] beschließen.“

113 Rs. C-366/99 (Joseph Griesmar ./. Ministre de l'Économic, des Finances et de l'Industrie, Ministre de la Fonction publique, de la Réforme de l'État et de la Décentralisation), Urt. v. 29.11. 2001, Slg. 2001 , I-9383. 
restriktiv auslegt. ${ }^{114}$ Das Urteil betraf das Maastrichter Sozialprotokoll. Es ist jedoch kein Grund ersichtlich, weshalb der EuGH diese Argumentation nicht auch in Zukunft auf den dieser Vorschrift nachgebildeten Art. 141 Abs. 4 EG übertragen sollte.

Nach Ansicht des EuGH erlaubt diese Klausel nur solche Vergünstigungen, die „den Frauen helfen, ihr Berufsleben gleichberechtigt im Verhältnis zu den Männern zu führen“, und den Zweck verfolgen, „den Schwierigkeiten abzuhelfen, auf die sie während ihrer beruflichen Laufbahn stoßen können“. ${ }^{115}$ Nicht erfasst seien hingegen Vorteile, die nach Beendigung des Berufslebens erlittene Nachteile ausgleichen sollen. Positive Folge dieser zeitlichen Differenzierung ist es, dass die Mitgliedstaaten gezwungen werden, sich auf Frauenfördermaßnahmen zu konzentrieren, die sofort, d. h. bereits im Arbeitsleben, wirken und nicht erst nachträglich, gewissermaßen als Schmerzensgeld für eine gesellschaftliche Realität - im entschiedenen Fall die Tatsache, dass in der Regel Frauen die maßgebliche Rolle bei der Kindererziehung spielen und die damit Karrierenachteile erfahren. Unmittelbare negative Folge dieser Rechtsprechung ist freilich, dass rentenrechtliche Besserstellungen von Frauen europarechtlich keinen Bestand haben und angesichts knapper öffentlicher Kassen die Bereitschaft zu und Möglichkeit von früher einsetzenden Kompensationsmechanismen gering sein dürften.

Mit diesen letzten Anmerkungen zur gerichtlichen Perspektive auf das Gemeinschaftsrecht komme ich zu meiner Antwort auf die eingangs gestellte Frage nach „Fortschritt oder Regression“ bei der Verankerung des Gleichstellungsgebotes auf Europaebene: Ich antworte mit einem entschiedenen „Das kommt darauf an“. Die textlichen Änderungen des EG-Vertrages, insbesondere durch den Amsterdamer Vertrag, sind zweifellos als Fortschritt anzusehen. Das gilt vor allem für die Anerkennung der Gleichstellung als allgemeines Ziel des Gemeinschaftsrechts und ihren hohen Rang durch die Querschnittsklausel. Auf der Ebene der Rechtsanwendung hat insbesondere die Rechtsprechung des EuGH mit den Fortschritten bei der Normierung des Gleichstellungsgebots jedoch nicht mitgehalten. Das Europarecht schreitet in der Tat unaufhörlich voran im Bereich der Gleichstellung jedoch nur in kleinen Schritten.

114 Vgl. Art. 6 Abs. 3 des Maastrichter Sozialprotokolls, a. a. O.

115 Rs. Griesmar, a. a. O., Rdnr. 64 und 65. 
